\title{
Combinatorics of exceptional sequences in type A
}

\author{
Alexander Garver* \\ Laboratoire de Combinatoire et d'Informatique Mathématique \\ Université du Québec à Montréal \\ Montréal, Canada \\ alexander.garver@lacim.ca \\ Kiyoshi Igusa ${ }^{\dagger}$ \\ Department of Mathematics \\ Brandeis University \\ Waltham, U.S.A. \\ igusa@brandeis.edu \\ Jacob P. Matherne \\ School of Mathematics \\ Institute for Advanced Study \\ Princeton, U.S.A. \\ matherne@math.ias.edu \\ Jonah Ostroff \\ Department of Mathematics \\ University of Washington \\ Seattle, U.S.A. \\ ostroff@math. washington.edu
}

Submitted: Jun 27, 2016; Accepted: Dec 5, 2018; Published: Feb 8, 2019

(C) The authors. Released under the CC BY-ND license (International 4.0).

\begin{abstract}
Exceptional sequences are certain sequences of quiver representations. We introduce a class of objects called strand diagrams and use these to classify exceptional sequences of representations of a quiver whose underlying graph is a type $\mathbb{A}_{n}$ Dynkin diagram. We also use variations of these objects to classify c-matrices of such quivers, to interpret exceptional sequences as linear extensions of explicitly constructed posets, and to give a simple bijection between exceptional sequences and certain saturated chains in the lattice of noncrossing partitions.
\end{abstract}

Mathematics Subject Classifications: 16G20, 05E10, 13F60

*The first author was supported by a Research Training Group, RTG Grant DMS-1148634.

${ }^{\dagger}$ The second author was supported by National Security Agency Grant H98230-13-1-0247.

$\ddagger$ The third author was supported by GAANN Grant P200A120001, an LSU Dissertation Year Fellowship, NSF Grant DMS-1638352, and the Association of Members of the Institute for Advanced Study (AMIAS). 


\section{Introduction}

Exceptional sequences are certain sequences of quiver representations with strong homological properties. They were first considered by Crawley-Boevey [CB93] and Ringel [Rin94]. Crawley-Boevey showed that the braid group acts transitively on the set of complete exceptional sequences (i.e., exceptional sequences of maximal length) [CB93]. This result was generalized to hereditary Artin algebras by Ringel [Rin94]. Since that time, they have been studied by Meltzer for weighted projective lines [Mel04], and by Araya for Cohen-Macaulay modules over one-dimensional graded Gorenstein rings with a simple singularity [Ara99]. Exceptional sequences have also been connected to many other areas of mathematics since their invention:

- chains in the lattice of noncrossing partitions [Bes03, HK16, IT09],

- c-matrices and cluster algebras [ST13],

- factorizations of Coxeter elements [IS10], and

- $t$-structures and derived categories [Bez03, BK89, Rud90].

Despite their ubiquity, very little work has been done to concretely describe exceptional sequences, even for path algebras of Dynkin quivers [Ara13, GM15]. In this paper, we give a concrete description of exceptional sequences for type $\mathbb{A}_{n}$ quivers with any orientation. This work extends and elaborates on a classification of exceptional sequences for the linearly-ordered quiver obtained in [GM15] by the first and third authors.

Exceptional sequences consist of indecomposable representations. For a quiver $Q$ of type $\mathbb{A}_{n}$, the indecomposable representations are completely determined by their dimension vectors, which are of the form

$$
(0, \ldots, 0,1, \ldots, 1,0, \ldots, 0) \in \mathbb{Z}_{\geqslant 0}^{n}
$$

Let us denote such a representation by $X_{i, j}^{\epsilon}$, where $\epsilon$ is a vector that keeps track of the orientation of the quiver, and $i+1$ and $j$ are the positions where the string of 1 's begins and ends, respectively.

This simple description allows us to view exceptional sequences as combinatorial objects. Define a map $\Phi_{\epsilon}$ which associates to each indecomposable representation $X_{i, j}^{\epsilon}$ a curve $\Phi_{\epsilon}\left(X_{i, j}^{\epsilon}\right)$ connecting two of $n+1$ points in $\mathbb{R}^{2}$. We will refer to such curves as strands. ${ }^{1}$

As exceptional sequences are certain collections of representations, the map $\Phi_{\epsilon}$ allows one to regard them as collections of strands.

The following lemma is the foundation for all of our results in this paper; it characterizes the homological data encoded by a pair of strands and thus by a pair of representations. A sequence of exceptional representations forms an exceptional sequence if

\footnotetext{
here.

${ }^{1}$ The curves $\Phi_{\epsilon}\left(X_{i, j}^{\epsilon}\right)$ will have some additional topological conditions (see Definition 5) that we omit
} 

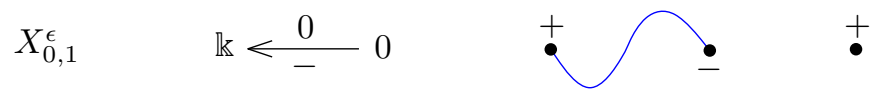

Figure 1: An example of the indecomposable representation $X_{0,1}^{\epsilon}$ on a type $\mathbb{A}_{2}$ quiver and the corresponding strand $\Phi_{\epsilon}\left(X_{0,1}^{\epsilon}\right)$.

and only if each of its subsequences of length two is exceptional. We refer to such length two exceptional sequences as exceptional pairs. Thus, Lemma 11, which we now state, allows us to completely classify exceptional sequences using strand diagrams.

Lemma 11. Let $Q_{\epsilon}$ be a type $\mathbb{A}$ Dynkin quiver. Fix two nonisomorphic indecomposable representations $U, V \in \operatorname{ind}\left(\operatorname{rep}_{\mathbb{k}}\left(Q_{\epsilon}\right)\right)$.

a) The strands $\Phi_{\epsilon}(U)$ and $\Phi_{\epsilon}(V)$ intersect nontrivially if and only if neither $(U, V)$ nor $(V, U)$ are exceptional pairs.

b) The strand $\Phi_{\epsilon}(U)$ is clockwise from $\Phi_{\epsilon}(V)$ if and only if $(U, V)$ is an exceptional pair and $(V, U)$ is not an exceptional pair.

c) The strands $\Phi_{\epsilon}(U)$ and $\Phi_{\epsilon}(V)$ do not intersect at any of their endpoints and they do not intersect nontrivially if and only if $(U, V)$ and $(V, U)$ are both exceptional pairs.

The paper is organized in the following way. In Section 2, we give the preliminaries on quivers and their representations which are needed for the rest of the paper.

In Section 3.1, we introduce strand diagrams. We show that exceptional collections (i.e., the underlying set of representations in an exceptional sequence) are classified by strand diagrams (see Theorem 12). Later, we decorate our strand diagrams by adding a label to each strand which endows the collection of strands with a linear order. We then show that our labeled diagrams classify exceptional sequences (Theorem 16) where the linear order on strands corresponds to the linear order on representations in the exceptional sequence. Although Lemma 11 is the main tool that allows us to obtain these results, we delay its proof until Section 3.2.

Next, we establish a connection between our work and c-matrices, which appear in the theory of cluster algebras. A c-matrix is an integer matrix that is produced by performing a sequence of quiver mutations on a certain type of ice quiver (see Section 2.1). The work of Speyer and Thomas (see [ST13]) gives a bijection between certain complete exceptional sequences of an arbitrary acyclic quiver and c-matrices arising from this quiver. In [Sei01], the number of complete exceptional sequences in type $\mathbb{A}_{n}$ is given (see also $\left[\mathrm{ONA}^{+} 13\right]$ for a systematic approach for all valued Dynkin quivers), and there are more of these than there are c-matrices. Thus, it is natural to ask which strand diagrams are describing $\mathbf{c}$-matrices. By establishing a bijection between the mixed cobinary trees of Igusa and Ostroff [IO13] and a certain collection of strand diagrams whose strands have an orientation, we give an answer to this question in Section 4. 
The conditions defining an exceptional collection give rise to the data of a poset on the representations in the exceptional collection. Moreover, the linear extensions of this poset are in bijection with the exceptional sequences that can be formed from the same exceptional collection. In Section 5, we characterize the family of posets defined by type A exceptional collections.

In Section 6, we present some applications of the theory in type $\mathbb{A}$. We give combinatorial proofs that any two reddening sequences produce isomorphic ice quivers (see [Kel12] for a general proof in all types using deep category-theoretic techniques) and that there is a bijection between exceptional sequences and certain saturated chains in the lattice of noncrossing partitions.

\section{Preliminaries}

We will be interested in the connection between exceptional sequences and the c-matrices of an acyclic quiver $Q$, so we begin by defining these. After that, we define quiver representations and exceptional sequences. We conclude this section by explaining the notation we will use to discuss exceptional representations of quivers that are orientations of a type $\mathbb{A}_{n}$ Dynkin diagram.

\subsection{Quiver mutation}

A quiver $Q$ is a directed graph. In other words, $Q$ is a 4-tuple $\left(Q_{0}, Q_{1}, s, t\right)$, where $Q_{0}=[m]:=\{1,2, \ldots, m\}$ is a set of vertices, $Q_{1}$ is a set of arrows, and two functions $s, t: Q_{1} \rightarrow Q_{0}$ defined so that for every $a \in Q_{1}$, we have $s(a) \stackrel{a}{\rightarrow} t(a)$. An ice quiver is a pair $(Q, F)$ with $Q$ a quiver and $F \subset Q_{0}$ a set of frozen vertices with the additional restriction that there are no two vertices $i, j \in F$ with an arrow of $Q$ connecting them. We refer to the elements of $Q_{0} \backslash F$ as mutable vertices. By convention, we assume $Q_{0} \backslash F=[n]$ and $F=[n+1, m]:=\{n+1, n+2, \ldots, m\}$. Any quiver $Q$ can be regarded as an ice quiver by setting $Q=(Q, \emptyset)$.

If $Q$ has no loops and no 2-cycles, one may define mutation of an ice quiver $(Q, F)$ as follows. The mutation of an ice quiver $(Q, F)$ at mutable vertex $k$, denoted $\mu_{k}$, produces a new ice quiver $\left(\mu_{k} Q, F\right)$ by the three-step process:

(1) For every 2-path $i \rightarrow k \rightarrow j$ in $Q$, adjoin a new arrow $i \rightarrow j$.

(2) Reverse the direction of all arrows incident to $k$ in $Q$.

(3) Remove a maximal collection of disjoint 2-cycles in the resulting quiver as well as all of the arrows between two frozen vertices.

We show an example of mutation below, depicting the mutable (resp., frozen) vertices in 
black (resp., blue).

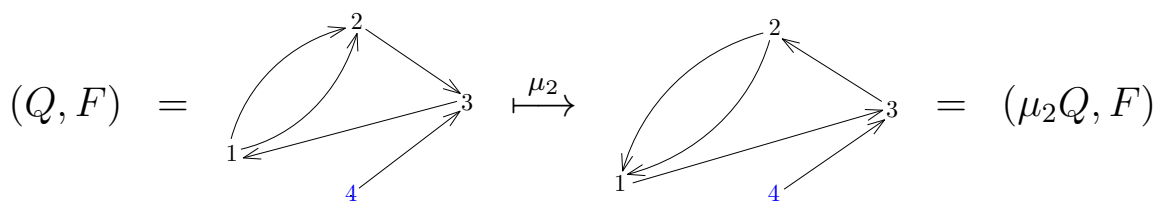

From now on, we will only consider quivers and ice quivers without loops and without 2-cycles. The information of such quivers and ice quivers can be equivalently described by its (skew-symmetric) exchange matrix. Given $(Q, F)$, we define $B=B_{(Q, F)}=$ $\left(b_{i j}\right) \in \mathbb{Z}^{n \times m}:=\{n \times m$ integer matrices $\}$ by $b_{i j}:=\#\left\{i \stackrel{a}{\rightarrow} j \in Q_{1}\right\}-\#\left\{j \stackrel{a}{\rightarrow} i \in Q_{1}\right\}$. Furthermore, ice quiver mutation can equivalently be defined as matrix mutation of the corresponding exchange matrix. Given an exchange matrix $B \in \mathbb{Z}^{n \times m}$, the mutation of $B$ at $k \in[n]$, also denoted $\mu_{k}$, produces a new exchange matrix $\mu_{k}(B)=\left(b_{i j}^{\prime}\right)$ with entries

$$
b_{i j}^{\prime}:=\left\{\begin{array}{cl}
-b_{i j} & : \text { if } i=k \text { or } j=k \\
b_{i j}+\frac{\left|b_{i k}\right| b_{k j}+b_{i k}\left|b_{k j}\right|}{2} & : \text { otherwise. }
\end{array}\right.
$$

For example, the mutation of the ice quiver above (here $m=4$ and $n=3$ ) translates into the following matrix mutation. Note that mutation of matrices (and of ice quivers) is an involution (i.e., $\mu_{k} \circ \mu_{k}(B)=B$ ).

$$
B_{(Q, F)}=\left[\begin{array}{ccc|c}
0 & 2 & -1 & 0 \\
-2 & 0 & 1 & 0 \\
1 & -1 & 0 & -1
\end{array}\right] \stackrel{\mu_{2}}{\longmapsto}\left[\begin{array}{ccc|r}
0 & -2 & 1 & 0 \\
2 & 0 & -1 & 0 \\
-1 & 1 & 0 & -1
\end{array}\right]=B_{\left(\mu_{2} Q, F\right)} .
$$

Given a quiver $Q$, we define its framed (resp., coframed) quiver to be the ice quiver $\widehat{Q}:=\left(Q^{\prime}, F\right)$ (resp., $\left.\check{Q}:=\left(Q^{\prime \prime}, F\right)\right)$ where $Q_{0}^{\prime}=Q_{0} \sqcup F\left(=Q_{0}^{\prime \prime}\right), F=[n+1,2 n]$, and $Q_{1}^{\prime}:=Q_{1} \sqcup\{i \rightarrow n+i: i \in[n]\}$ (resp., $Q_{1}^{\prime \prime}:=Q_{1} \sqcup\{n+i \rightarrow i: i \in[n]\}$ ). We define the exchange tree of $\widehat{Q}$, denoted $E T(\widehat{Q})$, to be the graph whose vertices are ice quivers obtained from $\widehat{Q}$ by a finite sequence of mutations without consecutive repetitions and with two vertices connected by an edge if and only if the corresponding quivers are obtained from each other by a single mutation. Similarly, define the exchange graph of $\widehat{Q}$, denoted $E G(\widehat{Q})$, to be the quotient of $E T(\widehat{Q})$ where two vertices are identified if and only if there is a frozen isomorphism of the corresponding quivers (i.e., an isomorphism that fixes the frozen vertices). Such an isomorphism is equivalent to a simultaneous permutation of the rows and columns of the corresponding exchange matrices.

Given $\widehat{Q}$, we define the c-matrix $C=C_{R}$ (resp., $\bar{C}=\bar{C}_{R}$ ) of $R \in E T(\widehat{Q})$ (resp., $R \in E G(\widehat{Q}))$ to be the submatrix of $B_{R}$ where $C:=\left(b_{i j}\right)_{i \in[n], j \in[n+1,2 n]}$ (resp., $\bar{C}:=$ $\left.\left(b_{i j}\right)_{i \in[n], j \in[n+1,2 n]}\right)$. We let c-mat $(Q):=\left\{\bar{C}_{R}: R \in E G(\widehat{Q})\right\}$. By definition, $B_{R}$ (resp., $\left.\bar{C}\right)$ is only defined up to simultaneous permutations of its rows and first $n$ columns (resp., up to permutations of its rows) for any $R \in E G(\widehat{Q})$.

A row vector of a c-matrix, $\vec{c}$, is known as a c-vector. The celebrated theorem of Derksen, Weyman, and Zelevinsky [DWZ10, Theorem 1.7], known as sign-coherence of 
c-vectors, states that for any $R \in E T(\widehat{Q})$ and $i \in[n]$ the c-vector $\overrightarrow{c_{i}}$ is a nonzero element of $\mathbb{Z}_{\geqslant 0}^{n}$ or $\mathbb{Z}_{\leqslant 0}^{n}$. In the former case, we say a c-vector is positive, and in the latter case, we say a c-vector is negative.

\subsection{Representations of quivers}

A representation $V=\left(\left(V_{i}\right)_{i \in Q_{0}},\left(\varphi_{a}\right)_{a \in Q_{1}}\right)$ of a quiver $Q$ is an assignment of a finite dimensional $\mathbb{k}$-vector space $V_{i}$ to each vertex $i$ and a $\mathbb{k}$-linear map $\varphi_{a}: V_{s(a)} \rightarrow V_{t(a)}$ to each arrow $a$ where $\mathbb{k}$ is a field. The dimension vector of $V$ is the vector $\operatorname{dim}(V):=$ $\left(\operatorname{dim} V_{i}\right)_{i \in Q_{0}}$. The support of $V$ is the set $\operatorname{supp}(V):=\left\{i \in Q_{0}: V_{i} \neq 0\right\}$. Here is an example of a representation, with $\underline{\operatorname{dim}}(V)=(2,1,3)$, of the mutable part of the quiver depicted in Section 2.1.

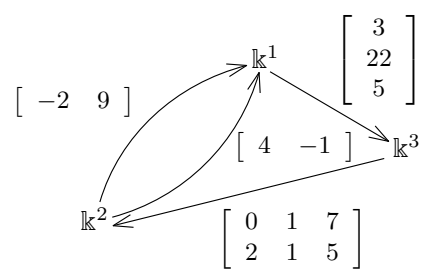

Let $V=\left(\left(V_{i}\right)_{i \in Q_{0}},\left(\varphi_{a}\right)_{a \in Q_{1}}\right)$ and $W=\left(\left(W_{i}\right)_{i \in Q_{0}},\left(\varrho_{a}\right)_{a \in Q_{1}}\right)$ be two representations of a quiver $Q$. A morphism $\theta: V \rightarrow W$ consists of a collection of linear maps $\theta_{i}: V_{i} \rightarrow W_{i}$ that are compatible with each of the linear maps in $V$ and $W$. That is, for each arrow $a \in Q_{1}$, we have $\theta_{t(a)} \circ \varphi_{a}=\varrho_{a} \circ \theta_{s(a)}$. An isomorphism of quiver representations is a morphism $\theta: V \rightarrow W$ where $\theta_{i}$ is a $\mathbb{k}$-vector space isomorphism for all $i \in Q_{0}$. We define $V \oplus W:=\left(\left(V_{i} \oplus W_{i}\right)_{i \in Q_{0}},\left(\varphi_{a} \oplus \varrho_{a}\right)_{a \in Q_{1}}\right)$ to be the direct sum of $V$ and $W$. We say that a nonzero representation $V$ is indecomposable if it is not isomorphic to a direct sum of two nonzero representations. Note that the representations of a quiver $Q$ along with morphisms between them form an abelian category, denoted by $\operatorname{rep}_{\mathbb{k}}(Q)$, with the indecomposable representations forming a full subcategory, denoted by ind $\left(\operatorname{rep}_{\mathbb{k}}(Q)\right)$.

We remark that representations of $Q$ can equivalently be regarded as modules over the path algebra $\mathbb{k} Q$. As such, one can define $\operatorname{Ext}_{\mathbb{k} Q}^{s}(V, W)$ for $s \geqslant 1$ and $\operatorname{Hom}_{\mathbb{k} Q}(V, W)$ for any representations $V$ and $W$, and $\operatorname{Hom}_{\mathbb{k} Q}(V, W)$ is isomorphic to the vector space of all morphisms $\theta: V \rightarrow W$. We refer the reader to [ASS06] for more details on representations of quivers.

An exceptional representation $V_{j}$ of $Q$ is a representation where $\operatorname{End}_{\mathbb{k} Q}\left(V_{j}\right)$ is a division algebra and $\operatorname{Ext}_{\mathbb{k} Q}^{s}\left(V_{j}, V_{j}\right)=0$ for all $s \geqslant 1$. Any exceptional representation is indecomposable, but not all indecomposable representations are exceptional. For Dynkin quivers, it is well-known that a representation is exceptional if and only if it is indecomposable. An exceptional sequence $\xi=\left(V_{1}, \ldots, V_{k}\right)$ is a sequence of exceptional representations satisfying $\operatorname{Hom}_{\mathbb{k} Q}\left(V_{j}, V_{i}\right)=0$ and $\operatorname{Ext}_{\mathbb{k} Q}^{s}\left(V_{j}, V_{i}\right)=0$ for all $s \geqslant 1$ if $i<j$. We use the term exceptional pair to mean an exceptional sequence consisting of exactly two exceptional representations. We define an exceptional collection $\bar{\xi}=\left\{V_{1}, \ldots, V_{k}\right\}$ to be a set of exceptional representations $V_{j}$ of $Q$ that can be ordered in such a way that they define an exceptional sequence. It was shown in [CB93] that the length $k$ of an exceptional sequence $\xi$ satisfies $k \leqslant n:=\# Q_{0}$; so when $k=n$, we say $\xi$ (resp., $\bar{\xi}$ ) is 
a complete exceptional sequence (CES) (resp., complete exceptional collection $(\mathrm{CEC}))$.

The following result of Speyer and Thomas gives a beautiful connection between cmatrices of an acyclic quiver $Q$ and CESs. It serves as motivation for our work. Before stating it we remark that for any $R \in E T(\widehat{Q})$ and any $i \in[n]$ where $Q$ is an acyclic quiver, the c-vector $\overrightarrow{c_{i}}=\overrightarrow{c_{i}}(R)= \pm \underline{\operatorname{dim}}\left(V_{i}\right)$ for some exceptional representation of $Q$ (see $[\mathrm{NC15}]$ or $[\mathrm{ST} 13])$.

Notation 1. Let $\vec{c}$ be a c-vector of an acyclic quiver $Q$. Define

$$
|\vec{c}|:=\left\{\begin{aligned}
\vec{c} & : \text { if } \vec{c} \text { is positive } \\
-\vec{c} & : \text { if } \vec{c} \text { is negative. }
\end{aligned}\right.
$$

Theorem 2 ([ST13]). Let $\bar{C} \in \boldsymbol{c}$-mat $(Q)$, let $\left\{\vec{c}_{i}\right\}_{i \in[n]}$ denote the c-vectors of $\bar{C}$, and let $\left|\overrightarrow{c_{i}}\right|=\underline{\operatorname{dim}}\left(V_{i}\right)$ for some exceptional representation of $Q$. There exists a permutation $\sigma \in \mathfrak{S}_{n}$ such that $\left(V_{\sigma(1)}, \ldots, V_{\sigma(n)}\right)$ is a CES with the property that if there exist positive $\boldsymbol{c}$-vectors in $\bar{C}$, then there exists $k \in[n]$ such that $\overrightarrow{c_{\sigma(i)}}$ is positive if and only if $i \in[k, n]$, and $\operatorname{Hom}_{\mathbb{k} Q}\left(V_{j}, V_{j^{\prime}}\right)=0$ for any $\overrightarrow{c_{j}}, \overrightarrow{c_{j^{\prime}}}$ that have the same sign. Conversely, any set of $n$ vectors $\overrightarrow{c_{1}}, \ldots, \overrightarrow{c_{n}}$ having these properties defines a $\boldsymbol{c}$-matrix whose row vectors are $\left\{\overrightarrow{c_{i}}\right\}_{i \in[n]}$.

\subsection{Quivers of type $\mathbb{A}_{n}$}

For the purposes of this paper, we will only be concerned with quivers of type $\mathbb{A}_{n}$. We say a quiver $Q$ is of type $\mathbb{A}_{n}$ if the underlying graph of $Q$ is a Dynkin diagram of type $\mathbb{A}_{n}$. By convention, two vertices $i$ and $j$ with $i<j$ in a type $\mathbb{A}_{n}$ quiver $Q$ are connected by an arrow if and only if $j=i+1$ and $i \in[n-1]$.

It will be convenient to denote a given type $\mathbb{A}_{n}$ quiver $Q$ using the notation $Q_{\epsilon}$, which we now define. Let $\epsilon=\left(\epsilon_{0}, \epsilon_{1}, \ldots, \epsilon_{n}\right) \in\{+,-\}^{n+1}$ and for $i \in[n-1]$ define $a_{i}^{\epsilon_{i}} \in Q_{1}$ by

$$
a_{i}^{\epsilon_{i}}:=\left\{\begin{array}{lll}
i \leftarrow i+1 & : & \epsilon_{i}=- \\
i \rightarrow i+1 & : & \epsilon_{i}=+
\end{array}\right.
$$

Then $Q_{\epsilon}:=\left(\left(Q_{\epsilon}\right)_{0}:=[n],\left(Q_{\epsilon}\right)_{1}:=\left\{a_{i}^{\epsilon_{i}}\right\}_{i \in[n-1]}\right)=Q$. One observes that the values of $\epsilon_{0}$ and $\epsilon_{n}$ do not affect $Q_{\epsilon}$.

Example 3. Let $n=5$ and $\epsilon=(-,+,-,+,-,+)$ so that $Q_{\epsilon}=1 \stackrel{a_{1}^{+}}{\longrightarrow} 2 \stackrel{a_{2}^{-}}{\longleftarrow} 3 \stackrel{a_{3}^{+}}{\longrightarrow} 4 \stackrel{a_{4}^{-}}{\longleftarrow}$ 5. Below we show its framed quiver $\widehat{Q}_{\epsilon}$.

$$
\widehat{Q}_{\epsilon}=\begin{array}{ccccc}
6 & 7 & 8 & 9 & 10 \\
\uparrow & \uparrow & \uparrow & \uparrow & \uparrow \\
1 \longrightarrow 2< & 3 \longrightarrow & 4< & 5
\end{array}
$$

Let $Q_{\epsilon}$ be given where $\epsilon=\left(\epsilon_{0}, \epsilon_{1}, \ldots, \epsilon_{n}\right) \in\{+,-\}^{n+1}$. Let $i, j \in[0, n]:=\{0,1, \ldots, n\}$ where $i<j$ and let $X_{i, j}^{\epsilon}=\left(\left(V_{\ell}\right)_{\ell \in\left(Q_{\epsilon}\right)_{0}},\left(\varphi_{a}^{i, j}\right)_{a \in\left(Q_{\epsilon}\right)_{1}}\right) \in \operatorname{rep}_{\mathbb{k}}\left(Q_{\epsilon}\right)$ be the indecomposable representation defined by 


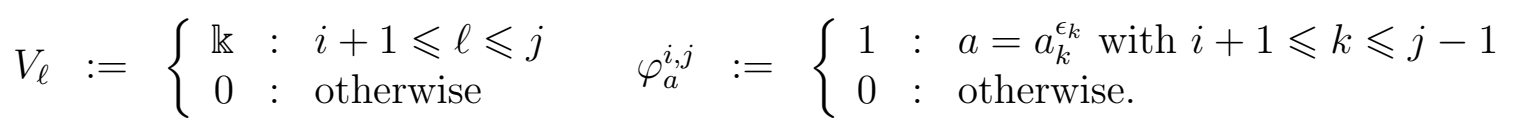

The objects of $\operatorname{ind}\left(\operatorname{rep}_{\mathrm{kk}}\left(Q_{\epsilon}\right)\right)$ are those of the form $X_{i, j}^{\epsilon}$ where $0 \leqslant i<j \leqslant n$, up to isomorphism.

Remark 4. If $X_{i, j}^{\epsilon}$ and $X_{k, \ell}^{\epsilon}$ are nonisomorphic indecomposables of $\operatorname{rep}_{\mathbb{k}}\left(Q_{\epsilon}\right)$, then we have that $\operatorname{Hom}_{\mathbb{k} Q_{\epsilon}}\left(X_{i, j}^{\epsilon}, X_{k, \ell}^{\epsilon}\right)=0$ or $\operatorname{Hom}_{\mathbb{k} Q_{\epsilon}}\left(X_{k, \ell}^{\epsilon}, X_{i, j}^{\epsilon}\right)=0$. This follows from the well-known fact that the Auslander-Reiten quiver of $\mathbb{k} Q_{\epsilon}$ is acyclic.

\section{Strand diagrams}

In this section, we define three different types of combinatorial objects: strand diagrams, labeled strand diagrams, and oriented strand diagrams. We will use these objects to classify exceptional collections, exceptional sequences, and c-matrices of a given type $\mathbb{A}_{n}$ quiver $Q_{\epsilon}$, so we fix such a quiver $Q_{\epsilon}$.

\subsection{Exceptional sequences and strand diagrams}

Let $\mathcal{S}_{n, \epsilon}:=\left\{\left(x_{0}, 0\right),\left(x_{1}, 0\right), \ldots,\left(x_{n}, 0\right)\right\} \subset \mathbb{R}^{2}$ be a collection of $n+1$ points arranged on the positive $x$-axis from left to right, i.e., $0<x_{0}<x_{1}<\cdots<x_{n}$, together with the function $\epsilon: \mathcal{S}_{n, \epsilon} \rightarrow\{+,-\}$ sending $\left(x_{i}, 0\right) \mapsto \epsilon_{i}$.

Definition 5. Let $i, j \in[0, n]$ where $i \neq j$. A strand $c(i, j)=c(j, i)$ on $\mathcal{S}_{n, \epsilon}$ is an isotopy class of simple curves in $\mathbb{R}^{2}$ where any $\gamma \in c(i, j)$ satisfies:

a) the endpoints of $\gamma$ are $\left(x_{i}, 0\right)$ and $\left(x_{j}, 0\right)$,

b) as a subset of $\mathbb{R}^{2}, \gamma \subset\left\{(x, y) \in \mathbb{R}^{2}: x_{\min (i, j)} \leqslant x \leqslant x_{\max (i, j)}\right\} \backslash\left\{\left(x_{k}, 0\right): x_{\min (i, j)}<\right.$ $\left.x_{k}<x_{\max (i, j)}\right\}$,

c) if $\min (i, j) \leqslant k \leqslant \max (i, j)$ and $\epsilon_{k}=+$ (resp., $\epsilon_{k}=-$ ), then $\gamma$ is locally below (resp., locally above) $\left(x_{k}, 0\right)$.

By locally below (resp., locally above) $\left(x_{k}, 0\right)$, we mean that for a given parameterization of $\gamma=\left(\gamma^{(1)}, \gamma^{(2)}\right):[0,1] \rightarrow \mathbb{R}^{2}$ there exists $\delta \in \mathbb{R}$ where

$$
0<\delta<\frac{1}{2} \min \left\{\left|x_{k}-x_{k-1}\right|,\left|x_{k}-x_{k+1}\right|\right\}
$$

such that $\gamma$ satisfies $\gamma^{(2)}(t)<0$ if $\epsilon_{k}=+\left(\right.$ resp., $\gamma^{(2)}(t)>0$ if $\left.\epsilon_{k}=-\right)$ for all $t \in(0,1)$ where $\gamma^{(1)}(t) \in\left(x_{k}-\delta, x_{k}+\delta\right)$.

There is a natural bijection $\Phi_{\epsilon}$ from the objects of ind $\left(\operatorname{rep}_{\mathbb{k}}\left(Q_{\epsilon}\right)\right)$ to the set of strands on $\mathcal{S}_{n, \epsilon}$ given by $\Phi_{\epsilon}\left(X_{i, j}^{\epsilon}\right):=c(i, j)$ where $i<j$. 


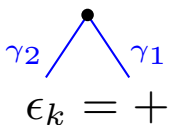

(a)

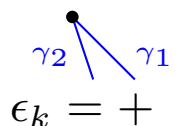

(b)

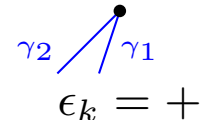

(c)

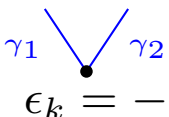

(d)

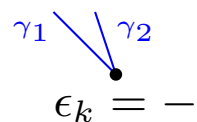

(e)

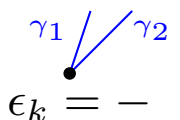

(f)

Figure 2: The six possible local configurations of strand $c\left(i_{2}, j_{2}\right)$ being clockwise from strand $c\left(i_{1}, j_{1}\right)$ near the shared endpoint $\left(x_{k}, 0\right)$.

Remark 6 . It is clear that any strand $c(i, j)$ can be represented by a monotone curve $\gamma \in c(i, j)$. That is, there exists a curve $\gamma \in c(i, j)$ with a parameterization $\gamma=\left(\gamma^{(1)}, \gamma^{(2)}\right)$ : $[0,1] \rightarrow \mathbb{R}^{2}$ such that if $t, s \in[0,1]$ and $t<s$, then $\gamma^{(1)}(t)<\gamma^{(1)}(s)$.

We say that two strands $c\left(i_{1}, j_{1}\right)$ and $c\left(i_{2}, j_{2}\right)$ intersect nontrivially if any two curves $\gamma_{\ell} \in c\left(i_{\ell}, j_{\ell}\right)$ with $\ell \in\{1,2\}$ intersect in their interiors. Otherwise, we say that $c\left(i_{1}, j_{1}\right)$ and $c\left(i_{2}, j_{2}\right)$ do not intersect nontrivially. For example, in the case where $n \geqslant 4$, $c(1,3), c(2,4)$ intersect nontrivially if and only if $\epsilon_{2}=\epsilon_{3}$. If $c\left(i_{1}, j_{1}\right)$ and $c\left(i_{2}, j_{2}\right)$ do not intersect nontrivially, we say that $c\left(i_{2}, j_{2}\right)$ is clockwise from $c\left(i_{1}, j_{1}\right)$ (or, equivalently, $c\left(i_{1}, j_{1}\right)$ is counterclockwise from $\left.c\left(i_{2}, j_{2}\right)\right)$ if and only if some $\gamma_{1} \in c\left(i_{1}, j_{1}\right)$ and $\gamma_{2} \in$ $c\left(i_{2}, j_{2}\right)$ share an endpoint $\left(x_{k}, 0\right)$, do not intersect in their interiors, and locally appear in one of the six configurations in Figure 2 preserving the property that $\gamma_{1} \in c\left(i_{1}, j_{1}\right)$ and $\gamma_{2} \in c\left(i_{2}, j_{2}\right)$.

Remark 7. Examination of these six diagrams in Figure 2 shows that the property of $c\left(i_{2}, j_{2}\right)$ being clockwise from $c\left(i_{1}, j_{1}\right)$ (or, equivalently, $c\left(i_{1}, j_{1}\right)$ being counterclockwise from $\left.c\left(i_{2}, j_{2}\right)\right)$ is well-defined. In fact:

(1) If $i_{1}<j_{1}=i_{2}<j_{2}$ then $c\left(i_{2}, j_{2}\right)$ is clockwise from $c\left(i_{1}, j_{1}\right)$ if and only if $\epsilon_{j_{1}}=-$ (Figure 2 (d) illustrates this, but Figure 2 (a) does not).

(2) If $i_{1}=i_{2}<j_{1}<j_{2}$ then $c\left(i_{2}, j_{2}\right)$ is clockwise from $c\left(i_{1}, j_{1}\right)$ if and only if $\epsilon_{j_{1}}=+$ (Figure 2 (b) and Figure 2 (f) both illustrate this).

(3) If $i_{1}<i_{2}<j_{1}=j_{2}$ then $c\left(i_{2}, j_{2}\right)$ is clockwise from $c\left(i_{1}, j_{1}\right)$ if and only if $\epsilon_{i_{2}}=+$. (Figure 2 (c) and Figure 2 (e) both illustrate this).

A given collection of strands $d=\left\{c\left(i_{\ell}, j_{\ell}\right)\right\}_{\ell \in[k]}$ with $k \leqslant n$, naturally defines a graph with vertex set $\mathcal{S}_{n, \epsilon}$ and edge set $\left\{\left\{\left(x_{s}, 0\right),\left(x_{t}, 0\right)\right\}: c(s, t) \in d\right\}$. We refer to this graph as the graph determined by $d$.

Remark 8. Any monotone curve $\gamma \in c(i, j)$ is the graph of a continuous function $f_{\gamma}$ : $\left[x_{i}, x_{j}\right] \rightarrow \mathbb{R}$ having the following properties:

1. $f_{\gamma}\left(x_{i}\right)=0=f_{\gamma}\left(x_{j}\right)$,

2. $f_{\gamma}\left(x_{k}\right)<0$ for all $i<k<j$ with $\epsilon_{k}=+$, and

3. $f_{\gamma}\left(x_{\ell}\right)>0$ for all $i<\ell<j$ with $\epsilon_{\ell}=-$. 
Conversely, any such continuous function is the graph of a monotone curve in $c(i, j)$.

Definition 9. A strand diagram $d=\left\{c\left(i_{\ell}, j_{\ell}\right)\right\}_{\ell \in[k]}$ on $\mathcal{S}_{n, \epsilon}$ where $k \leqslant n$ is a collection of strands on $\mathcal{S}_{n, \epsilon}$ that satisfies the following conditions:

a) distinct strands do not intersect nontrivially, and

b) the graph determined by $d$ is a forest (i.e., a disjoint union of trees).

Let $\mathcal{D}_{k, \epsilon}$ denote the set of strand diagrams on $\mathcal{S}_{n, \epsilon}$ with $k$ strands, and let $\mathcal{D}_{\epsilon}$ denote the set of all strand diagrams on $\mathcal{S}_{n, \epsilon}$. This implies that

$$
\mathcal{D}_{\epsilon}=\bigsqcup_{k \in[n]} \mathcal{D}_{k, \epsilon} .
$$

Example 10. Let $n=4$ and $\epsilon=(-,+,-,+,+)$ so that $Q_{\epsilon}=1 \stackrel{a_{1}^{+}}{\longrightarrow} 2 \stackrel{a_{2}^{-}}{\longleftarrow} 3 \stackrel{a_{3}^{+}}{\longrightarrow} 4$. Then we have that $d_{1}=\{c(0,1), c(0,2), c(2,3), c(2,4)\} \in \mathcal{D}_{4, \epsilon}$ and $d_{2}=\{c(0,4), c(1,3), c(2,4)\}$ $\in \mathcal{D}_{3, \epsilon}$. We draw these strand diagrams below.

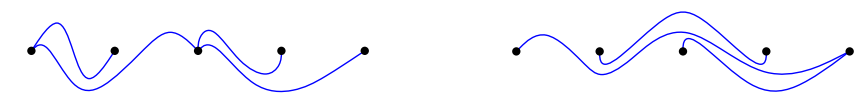

The following technical lemma classifies when two nonisomorphic indecomposable representations of $Q_{\epsilon}$ define zero, one, or two exceptional pairs. Its proof appears in Section 3.2 .

Lemma 11. Let $Q_{\epsilon}$ be given. Fix two nonisomorphic indecomposable representations $U, V \in \operatorname{ind}\left(r e p_{\mathbb{l k}}\left(Q_{\epsilon}\right)\right)$.

a) The strands $\Phi_{\epsilon}(U)$ and $\Phi_{\epsilon}(V)$ intersect nontrivially if and only if neither $(U, V)$ nor $(V, U)$ are exceptional pairs.

b) The strand $\Phi_{\epsilon}(U)$ is clockwise from $\Phi_{\epsilon}(V)$ if and only if $(U, V)$ is an exceptional pair and $(V, U)$ is not an exceptional pair.

c) The strands $\Phi_{\epsilon}(U)$ and $\Phi_{\epsilon}(V)$ do not intersect at any of their endpoints and they do not intersect nontrivially if and only if $(U, V)$ and $(V, U)$ are both exceptional pairs.

Furthermore, there exist monotone curves $\gamma_{\epsilon}(U) \in \Phi_{\epsilon}(U)$ for all $U \in \operatorname{ind}\left(\operatorname{rep}_{\mathbb{k}}\left(Q_{\epsilon}\right)\right)$ so that $\gamma_{\epsilon}(U), \gamma_{\epsilon}(V)$ have a unique crossing, have a common endpoint, or have no point of intersection, respectively, if and only if $U$ and $V$ satisfy $a), b)$, or c), respectively.

Using Lemma 11 we obtain our first main result. The following theorem says that the data of an exceptional collection is completely encoded in the strand diagram it defines.

Theorem 12. Let $\overline{\mathcal{E}}_{\epsilon}:=\left\{\right.$ exceptional collections of $\left.Q_{\epsilon}\right\}$. There is a bijection $\overline{\mathcal{E}}_{\epsilon} \rightarrow \mathcal{D}_{\epsilon}$ defined by

$$
\bar{\xi}_{\epsilon}=\left\{X_{i_{\ell}, j_{\ell}}^{\epsilon}\right\}_{\ell \in[k]} \mapsto\left\{c\left(i_{\ell}, j_{\ell}\right)\right\}_{\ell \in[k]} .
$$


Proof. It is enough to show that the image of $\overline{\mathcal{E}}_{\epsilon}$ under this map is a subset of $\mathcal{D}_{\epsilon}$ and this map has a well-defined inverse.

Let $\bar{\xi}_{\epsilon}=\left\{X_{i_{\ell}, j_{\ell}}^{\epsilon}\right\}_{\ell \in[k]}$ be an exceptional collection of $Q_{\epsilon}$. Let $\xi_{\epsilon}$ be an exceptional sequence obtained from $\bar{\xi}_{\epsilon}$ by choosing a suitable ordering of its representations. Without loss of generality, assume $\xi_{\epsilon}=\left(X_{i_{\ell}, j_{\ell}}^{\epsilon}\right)_{\ell \in[k]}$ is an exceptional sequence. Thus, $\left(X_{i_{\ell}, j_{\ell}}^{\epsilon}, X_{i_{p}, j_{p}}^{\epsilon}\right)$ is an exceptional pair for all $\ell$ and $p$ satisfying $\ell<p$. Lemma 11 a) implies that distinct strands of $\left\{c\left(i_{\ell}, j_{\ell}\right)\right\}_{\ell \in[k]}$ do not intersect nontrivially. This implies that the curves $\gamma_{\ell}=$ $\gamma_{\epsilon}\left(X_{i_{\ell}, j_{\ell}}^{\epsilon}\right) \in c\left(i_{\ell}, j_{\ell}\right)$ given in Lemma 11 will be pairwise nonintersecting.

Now we will show that $\left\{c\left(i_{\ell}, j_{\ell}\right)\right\}_{\ell \in[k]}$ has no cycles. Suppose that $c\left(i_{\ell_{1}}, j_{\ell_{1}}\right), \ldots$, $c\left(i_{\ell_{p}}, j_{\ell_{p}}\right)$ is a cycle of length $p \leqslant k$ in $\left\{c\left(i_{\ell}, j_{\ell}\right)\right\}_{\ell \in[k]}$. Since the cycle is embedded in the plane, it encloses one region. Choose the orientation of the cycle so that it goes clockwise around this region. Interpreting the indices cyclically, we have that $c\left(i_{\ell_{s}}, j_{\ell_{s}}\right)$ is clockwise from $c\left(i_{\ell_{s+1}}, j_{\ell_{s+1}}\right)$ for all $s \in[p]$. Therefore $X_{i_{\ell_{s}}, j_{\ell_{s}}}^{\epsilon}$ precedes $X_{i_{\ell_{s+1}}, j_{\ell_{s+1}}}$ in the exceptional sequence for all $s \in[p]$, again interpreting the indices cyclically. But this is impossible. This contradicts the fact that $\left(X_{i_{\ell}, j_{\ell}}^{\epsilon}\right)_{\ell \in[k]}$ is an exceptional sequence. Hence, the graph determined by $\left\{c\left(i_{\ell}, j_{\ell}\right)\right\}_{\ell \in[k]}$ is a tree. We have shown that $\left\{c\left(i_{\ell}, j_{\ell}\right)\right\}_{\ell \in[k]} \in \mathcal{D}_{k, \epsilon}$.

Now let $d=\left\{c\left(i_{\ell}, j_{\ell}\right)\right\}_{\ell \in[k]} \in \mathcal{D}_{k, \epsilon}$. Since $c\left(i_{\ell}, j_{\ell}\right)$ and $c\left(i_{m}, j_{m}\right)$ do not intersect nontrivially, it follows that $\left(\Phi_{\epsilon}^{-1}\left(c\left(i_{\ell}, j_{\ell}\right)\right), \Phi_{\epsilon}^{-1}\left(c\left(i_{m}, j_{m}\right)\right)\right)$ or $\left(\Phi_{\epsilon}^{-1}\left(c\left(i_{m}, j_{m}\right)\right), \Phi_{\epsilon}^{-1}\left(c\left(i_{\ell}, j_{\ell}\right)\right)\right)$ is an exceptional pair for every $\ell \neq m$. Notice that there exists $c\left(i_{\ell_{1}}, j_{\ell_{1}}\right) \in d$ such that $\left(\Phi_{\epsilon}^{-1}\left(c\left(i_{\ell_{1}}, j_{\ell_{1}}\right)\right), \Phi_{\epsilon}^{-1}\left(c\left(i_{\ell}, j_{\ell}\right)\right)\right)$ is an exceptional pair for every $c\left(i_{\ell}, j_{\ell}\right) \in d \backslash\left\{c\left(i_{\ell_{1}}, j_{\ell_{1}}\right)\right\}$. This is true because if such $c\left(i_{\ell_{1}}, j_{\ell_{1}}\right)$ did not exist, then $d$ must have a cycle. Set $E_{1}=\Phi_{\epsilon}^{-1}\left(c\left(i_{\ell_{1}}, j_{\ell_{1}}\right)\right)$. Now, choose $c\left(i_{\ell_{p}}, j_{\ell_{p}}\right)$ such that $\left(\Phi_{\epsilon}^{-1}\left(c\left(i_{\ell_{p}}, j_{\ell_{p}}\right)\right), \Phi_{\epsilon}^{-1}\left(c\left(i_{\ell}, j_{\ell}\right)\right)\right)$ is an exceptional pair for every $c\left(i_{\ell}, j_{\ell}\right) \in d \backslash\left\{c\left(i_{\ell_{1}}, j_{\ell_{1}}\right), \ldots, c\left(i_{\ell_{p}}, j_{\ell_{p}}\right)\right\}$ inductively and put $E_{p}=\Phi_{\epsilon}^{-1}\left(c\left(i_{\ell_{p}}, j_{\ell_{p}}\right)\right)$. By construction, $\left(E_{1}, \ldots, E_{k}\right)$ is an exceptional sequence, as desired.

Remark 13. This version of the argument in the third paragraph of the proof of Theorem 12 above was communicated to us by the anonymous referee, to whom we are grateful.

Our next step is to add distinct integer labels to each strand in a given strand diagram $d$. When these labels are a good labeling, they will describe exactly the order in which to put the representations corresponding to strands of $d$ so that the resulting sequence of representations is an exceptional sequence.

Definition 14. A labeled diagram $\hat{d}=\left\{\left(c\left(i_{\ell}, j_{\ell}\right), s_{\ell}\right)\right\}_{\ell \in[k]}$ on $\mathcal{S}_{n, \epsilon}$ is a set of pairs $\left(c\left(i_{\ell}, j_{\ell}\right), s_{\ell}\right)$ where $c\left(i_{\ell}, j_{\ell}\right)$ is a strand on $\mathcal{S}_{n, \epsilon}$ and $s_{\ell} \in[k]$ such that $d:=\left\{c\left(i_{\ell}, j_{\ell}\right)\right\}_{\ell \in[k]}$ is a strand diagram on $\mathcal{S}_{n, \epsilon}$ and $s_{\ell} \neq s_{\ell^{\prime}}$ for any distinct $\ell, \ell^{\prime} \in[k]$. We refer to the pairs $\left(c\left(i_{\ell}, j_{\ell}\right), s_{\ell}\right)$ as labeled strands and to $d$ as the underlying diagram of $\hat{d}$. We define the endpoints of a labeled strand $\left(c\left(i_{\ell}, j_{\ell}\right), s_{\ell}\right)$ to be the endpoints of $c\left(i_{\ell}, j_{\ell}\right)$.

Let $\left(x_{i}, 0\right) \in \mathcal{S}_{n, \epsilon}$ and let $\left(\left(c\left(i, j_{1}\right), s_{1}\right), \ldots,\left(c\left(i, j_{r}\right), s_{r}\right)\right)$ be the sequence of all labeled strands of $\hat{d}$ that have $\left(x_{i}, 0\right)$ as an endpoint, and assume that this sequence is ordered so that strand $c\left(i, j_{k}\right)$ is clockwise from $c\left(i, j_{k^{\prime}}\right)$ if $k^{\prime}<k$. We say the strand labeling of $\hat{d}$ is good if for each point $\left(x_{i}, 0\right) \in \mathcal{S}_{n, \epsilon}$ that is the endpoint of at least one labeled strand of $\hat{d}$, one has $s_{1}<\cdots<s_{r}$. Let $\hat{\mathcal{D}}_{k, \epsilon}$ denote the set of labeled strand diagrams on $\mathcal{S}_{n, \epsilon}$ with $k$ strands and with good strand labelings. 
Example 15. Let $n=4$ and $\epsilon=(-,+,-,+,+)$ so that $Q_{\epsilon}=1 \stackrel{a_{1}^{+}}{\longrightarrow} 2 \stackrel{a_{2}^{-}}{\longleftarrow} 3 \stackrel{a_{3}^{+}}{\longrightarrow} 4$. Below we show the labeled diagrams $\hat{d}_{1}=\{(c(0,1), 1),(c(0,2), 2),(c(2,3), 3),(c(2,4), 4)\}$ and $\hat{d}_{2}=\{(c(0,4), 1),(c(2,4), 2),(c(1,3), 3)\}$.

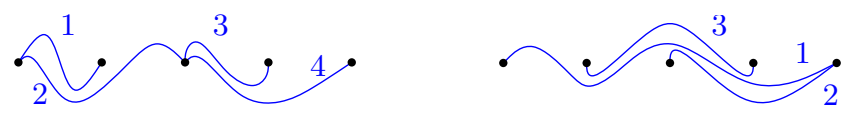

We have that $\hat{d}_{1} \in \hat{\mathcal{D}}_{4, \epsilon}$, but $\hat{d}_{2} \notin \hat{\mathcal{D}}_{3, \epsilon}$.

Theorem 16. Let $k \in[n]$ and let $\mathcal{E}_{\epsilon}:=\left\{\right.$ exceptional sequences of $Q_{\epsilon}$ of length $\left.k\right\}$. There is a bijection $\widetilde{\Phi}_{\epsilon}: \mathcal{E}_{\epsilon} \rightarrow \hat{\mathcal{D}}_{k, \epsilon}$ defined by

$$
\xi_{\epsilon}=\left(X_{i_{\ell}, j_{\ell}}^{\epsilon}\right)_{\ell \in[k]} \longmapsto\left\{\left(c\left(i_{\ell}, j_{\ell}\right), k+1-\ell\right)\right\}_{\ell \in[k]} .
$$

Proof. Let $\xi_{\epsilon} \in \mathcal{E}_{\epsilon}$. By Lemma 11 a), $\widetilde{\Phi}_{\epsilon}\left(\xi_{\epsilon}\right)$ has no strands that intersect nontrivially. Let $\left(V_{1}, V_{2}\right)$ be an exceptional pair appearing in $\xi_{\epsilon}$ with $V_{i}$ corresponding to strand $c_{i}$ in $\widetilde{\Phi}_{\epsilon}\left(\xi_{\epsilon}\right)$ for $i=1,2$, where $c_{1}$ and $c_{2}$ intersect only at one of their endpoints. Note that by the definition of $\widetilde{\Phi}_{\epsilon}$, the strand label of $c_{1}$ is larger than that of $c_{2}$. From Lemma $11 \mathrm{~b}$ ), strand $c_{1}$ is clockwise from $c_{2}$ in $\widetilde{\Phi}_{\epsilon}\left(\xi_{\epsilon}\right)$. Thus the strand labeling of $\widetilde{\Phi}_{\epsilon}\left(\xi_{\epsilon}\right)$ is good, so $\widetilde{\Phi}_{\epsilon}\left(\xi_{\epsilon}\right) \in \hat{\mathcal{D}}_{k, \epsilon}$ for any $\xi_{\epsilon} \in \mathcal{E}_{\epsilon}$.

Let $\widetilde{\Psi}_{\epsilon}: \hat{\mathcal{D}}_{k, \epsilon} \rightarrow \mathcal{E}_{\epsilon}$ be defined by $\left\{\left(c\left(i_{\ell}, j_{\ell}\right), \ell\right)\right\}_{\ell \in[k]} \mapsto\left(X_{i_{k}, j_{k}}^{\epsilon}, X_{i_{k-1}, j_{k-1}}^{\epsilon}, \ldots, X_{i_{1}, j_{1}}^{\epsilon}\right)$. We will show that $\widetilde{\Psi}_{\epsilon}(\hat{d}) \in \mathcal{E}_{\epsilon}$ for any $\hat{d} \in \hat{\mathcal{D}}_{k, \epsilon}$ and that $\widetilde{\Psi}_{\epsilon}=\widetilde{\Phi}_{\epsilon}^{-1}$. Let

$$
\widetilde{\Psi}_{\epsilon}\left(\left\{\left(c\left(i_{\ell}, j_{\ell}\right), \ell\right)\right\}_{\ell \in[k]}\right)=\left(X_{i_{k}, j_{k}}^{\epsilon}, X_{i_{k-1}, j_{k-1}}^{\epsilon}, \ldots, X_{i_{1}, j_{1}}^{\epsilon}\right) .
$$

Consider the pair $\left(X_{i_{s}, j_{s}}^{\epsilon}, X_{i_{s^{\prime}}, j_{s^{\prime}}}^{\epsilon}\right)$ with $s>s^{\prime}$. We will show that $\left(X_{i_{s}, j_{s}}^{\epsilon}, X_{i_{s^{\prime}}, j_{s^{\prime}}}^{\epsilon}\right)$ is an exceptional pair and thus conclude that $\widetilde{\Psi}_{\epsilon}\left(\left\{\left(c\left(i_{\ell}, j_{\ell}\right), \ell\right)\right\}_{\ell \in[k]}\right) \in \mathcal{E}_{\epsilon}$ for any $\hat{d} \in \hat{\mathcal{D}}_{k, \epsilon}$. Clearly, $c\left(i_{s}, j_{s}\right)$ and $c\left(i_{s^{\prime}}, j_{s^{\prime}}\right)$ do not intersect nontrivially. If $c\left(i_{s}, j_{s}\right)$ and $c\left(i_{s^{\prime}}, j_{s^{\prime}}\right)$ do not intersect at one of their endpoints, then by Lemma $11 \mathrm{c})\left(X_{i_{s}, j_{s}}^{\epsilon}, X_{i_{s^{\prime}}, j_{s^{\prime}}}^{\epsilon}\right)$ is exceptional. Now suppose $c\left(i_{s}, j_{s}\right)$ and $c\left(i_{s^{\prime}}, j_{s^{\prime}}\right)$ intersect at one of their endpoints. Because the strandlabeling of $\left\{\left(c\left(i_{\ell}, j_{\ell}\right), \ell\right)\right\}_{\ell \in[k]}$ is good, $c\left(i_{s}, j_{s}\right)$ is clockwise from $c\left(i_{s^{\prime}}, j_{s^{\prime}}\right)$. By Lemma 11 b), we have that $\left(X_{i_{s}, j_{s}}^{\epsilon}, X_{i_{s^{\prime}}, j_{s^{\prime}}}^{\epsilon}\right)$ is exceptional.

To see that $\widetilde{\Psi}_{\epsilon}=\widetilde{\Phi}_{\epsilon}^{-1}$, observe that

$$
\begin{aligned}
\widetilde{\Phi}_{\epsilon}\left(\widetilde{\Psi}_{\epsilon}\left(\left\{\left(c\left(i_{\ell}, j_{\ell}\right), \ell\right)\right\}_{\ell \in[k]}\right)\right) & =\widetilde{\Phi}_{\epsilon}\left(\left(X_{i_{k}, j_{k}}^{\epsilon}, X_{i_{k-1}, j_{k-1}}^{\epsilon}, \ldots, X_{i_{1}, j_{1}}^{\epsilon}\right)\right) \\
& =\left\{\left(c\left(i_{\ell}, j_{\ell}\right), k+1-(k+1-\ell)\right)\right\}_{\ell \in[k]} \\
& =\left\{\left(c\left(i_{\ell}, j_{\ell}\right), \ell\right)\right\}_{\ell \in[k]} .
\end{aligned}
$$

Thus $\widetilde{\Phi}_{\epsilon} \circ \widetilde{\Psi}_{\epsilon}=1_{\hat{\mathcal{D}}_{n, \epsilon}}$. Similarly, one shows that $\widetilde{\Psi}_{\epsilon} \circ \widetilde{\Phi}_{\epsilon}=1_{\mathcal{E}_{\epsilon}}$. Thus $\widetilde{\Phi}_{\epsilon}$ is a bijection.

The last combinatorial objects we discuss in this section are called oriented diagrams. These are strand diagrams whose strands have a direction. 
Definition 17. Let $\vec{c}\left(i_{\ell}, j_{\ell}\right)$ denote the data of the strand $c\left(i_{\ell}, j_{\ell}\right)$ on $\mathcal{S}_{n, \epsilon}$ and an orientation of each curve in $c\left(i_{\ell}, j_{\ell}\right)$ from $\left(x_{i_{\ell}}, 0\right)$ to $\left(x_{j_{\ell}}, 0\right)$. We refer to $\vec{c}\left(i_{\ell}, j_{\ell}\right)$ as an oriented strand on $\mathcal{S}_{n, \epsilon}$ and we define the endpoints of $\vec{c}\left(i_{\ell}, j_{\ell}\right)$ to be the endpoints of $c\left(i_{\ell}, j_{\ell}\right)$. An oriented diagram $\vec{d}=\left\{\vec{c}\left(i_{\ell}, j_{\ell}\right)\right\}_{\ell \in[k]}$ on $\mathcal{S}_{n, \epsilon}$ is a collection of oriented strands on $\mathcal{S}_{n, \epsilon}$ where $d=\left\{c\left(i_{\ell}, j_{\ell}\right)\right\}_{\ell \in[k]}$ is a strand diagram on $\mathcal{S}_{n, \epsilon}$. We refer to $d$ as the underlying diagram of $\vec{d}$.

Remark 18. When it is clear from the context what the values of $n$ and $\epsilon$ are, we will often refer to a strand diagram on $\mathcal{S}_{n, \epsilon}$ simply as a diagram. Similarly, we will often refer to labeled diagrams (resp., oriented diagrams) on $\mathcal{S}_{n, \epsilon}$ as labeled diagrams (resp., oriented diagrams). Additionally, if we have two diagrams $d_{1}$ and $d_{2}$ (both on $\mathcal{S}_{n, \epsilon}$ ) where $d_{1} \subset d_{2}$, we say that $d_{1}$ is a subdiagram of $d_{2}$. One analogously defines labeled subdiagrams (resp., oriented subdiagrams) of a labeled diagram (resp., oriented diagram).

We now define a special subset of the oriented diagrams on $\mathcal{S}_{n, \epsilon}$. As we will see, each element in this subset of oriented diagrams, denoted $\overrightarrow{\mathcal{D}}_{n, \epsilon}$, will correspond to a unique c-matrix $\bar{C} \in \mathbf{c}$-mat $\left(Q_{\epsilon}\right)$ and vice versa. Thus we obtain a diagrammatic classification of c-matrices (see Theorem 22).

Definition 19. Let $\overrightarrow{\mathcal{D}}_{n, \epsilon}$ denote the set of oriented diagrams $\vec{d}=\left\{\vec{c}\left(i_{\ell}, j_{\ell}\right)\right\}_{\ell \in[n]}$ on $\mathcal{S}_{n, \epsilon}$ with the property that for each $k \in[0, n]$ there exist integers $i_{1}, i_{2}, j \in[0, n]$ where $i_{1}<k<i_{2}$ and $j \in[0, n] \backslash\left\{i_{1}, k, i_{2}\right\}$ such that the oriented subdiagram $\overrightarrow{d_{k}}$ of $\vec{d}$ consisting of the oriented strands of $\vec{d}$ with $\left(x_{k}, 0\right)$ as an endpoint is an oriented subdiagram of one of the following two oriented diagrams on $\mathcal{S}_{n, \epsilon}$ :

i) $\overrightarrow{d_{+}}=\left\{\vec{c}\left(k, i_{1}\right), \vec{c}\left(k, i_{2}\right), \vec{c}(j, k)\right\}$ where $\epsilon_{k}=+($ shown in Figure 3 (left)) or

ii) $\overrightarrow{d_{-}}=\left\{\vec{c}\left(i_{1}, k\right), \vec{c}\left(i_{2}, k\right), \vec{c}(k, j)\right\}$ where $\epsilon_{k}=-($ shown in Figure 3 (right)).

Note that to make sense of the local pictures in Figure 3, we must also choose representatives of the strands in $\overrightarrow{d_{k}}$ which pairwise have no intersections in their interiors.
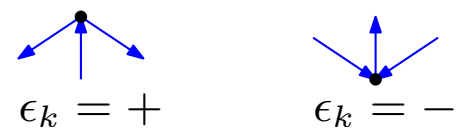

Figure 3: The oriented subdiagrams from Definition 19.

Lemma 20. Let $\left\{\vec{c}_{i}\right\}_{i \in[k]}$ be a collection of c-vectors of $Q_{\epsilon}$ where $k \leqslant n$. Let $\overrightarrow{c_{i}}=$ $\pm \underline{\operatorname{dim}}\left(X_{i_{1}, i_{2}}^{\epsilon}\right)$ where the sign is determined by $\overrightarrow{c_{i}}$. There is an injective map

$$
\left\{\begin{array}{c}
\text { noncrossing collections } \\
\text { of c-vectors }\left\{\overrightarrow{c_{i}}\right\}_{i \in[k]}
\end{array}\right\} \rightarrow\left\{\begin{array}{l}
\text { oriented diagrams } \\
\vec{d}=\left\{\vec{c}\left(i_{\ell}, j_{\ell}\right)\right\}_{\ell \in[k]}
\end{array}\right\}
$$


defined by

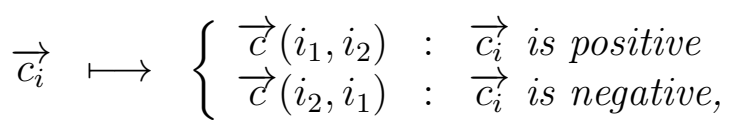

where $\left\{\vec{c}_{i}\right\}_{i \in[k]}$ is a noncrossing collection of c-vectors if $\Phi_{\epsilon}\left(X_{i_{1}, i_{2}}^{\epsilon}\right)$ and $\Phi_{\epsilon}\left(X_{i_{1}^{\prime}, i_{2}^{\prime}}^{\epsilon}\right)$ do not intersect nontrivially for any $i, i^{\prime} \in[k]$. In particular, each $\boldsymbol{c}$-matrix $\bar{C}_{\epsilon} \in \boldsymbol{c}$-mat $\left(Q_{\epsilon}\right)$ determines a unique oriented diagram denoted $\overrightarrow{d_{\bar{C}_{\epsilon}}}$ with $n$ oriented strands.

Example 21. Let $n=4$ and $\epsilon=(+,+,-,+,-)$ so that $Q_{\epsilon}=1 \stackrel{a_{1}^{+}}{\longrightarrow} 2 \stackrel{a_{2}^{-}}{\longleftarrow} 3 \stackrel{a_{3}^{+}}{\longrightarrow} 4$. After performing the mutation sequence $\mu_{3} \circ \mu_{2}$ to the corresponding framed quiver, we have the c-matrix with its oriented diagram.

$$
\left[\begin{array}{cccc}
1 & 1 & 0 & 0 \\
0 & 0 & 1 & 0 \\
0 & -1 & -1 & 0 \\
0 & 0 & 0 & 1
\end{array}\right]
$$

The following theorem shows that oriented diagrams belonging to $\overrightarrow{\mathcal{D}}_{n, \epsilon}$ are in bijection with c-matrices of $Q_{\epsilon}$. We delay its proof until Section 4 because it makes use of the concept of a mixed cobinary tree.

Theorem 22. The map c-mat $\left(Q_{\epsilon}\right) \rightarrow \overrightarrow{\mathcal{D}}_{n, \epsilon}$ induced by the map defined in Lemma 20 is a bijection.

\subsection{Proof of Lemma 11}

The proof of Lemma 11 requires some notions from representation theory of finite dimensional algebras, which we now briefly review. For a more comprehensive treatment of the following notions, we refer the reader to [ASS06].

Definition 23. Given a quiver $Q$ with $\# Q_{0}=n$, the Euler characteristic $($ of $Q$ ) is the $\mathbb{Z}$-bilinear (nonsymmetric) form $\mathbb{Z}^{n} \times \mathbb{Z}^{n} \rightarrow \mathbb{Z}$ defined by

$$
\langle\underline{\operatorname{dim}}(V), \underline{\operatorname{dim}}(W)\rangle=\sum_{i \geqslant 0}(-1)^{i} \operatorname{dim} \operatorname{Ext}_{\mathbb{k} Q}^{i}(V, W)
$$

for every $V, W \in \operatorname{rep}_{\mathbb{k}}(Q)$.

For hereditary algebras $A$ (e.g., path algebras of acyclic quivers), $\operatorname{Ext}_{A}^{i}(V, W)=0$ for $i \geqslant 2$ and the formula reduces to

$$
\langle\underline{\operatorname{dim}}(V), \underline{\operatorname{dim}}(W)\rangle=\operatorname{dim} \operatorname{Hom}_{\mathbb{k} Q}(V, W)-\operatorname{dim}_{\operatorname{Ext}_{\mathbb{k} Q}^{1}(V, W) .}
$$

The following lemma, which appears in [ASS06], gives a simple formula for the Euler characteristic. 
Lemma 24. Given an acyclic quiver $Q$ with $\# Q_{0}=n$ and vectors $x=\left(x_{1}, x_{2}, \ldots, x_{n}\right)$, $y=\left(y_{1}, y_{2}, \ldots, y_{n}\right) \in \mathbb{Z}^{n}$, the Euler characteristic of $Q$ has the form

$$
\langle x, y\rangle=\sum_{i \in Q_{0}} x_{i} y_{i}-\sum_{\alpha \in Q_{1}} x_{s(\alpha)} y_{t(\alpha)}
$$

Next, we give a slight simplification of the previous formula. Recall that the support of $V \in \operatorname{rep}_{\mathbb{k}}(Q)$ is the set $\operatorname{supp}(V):=\left\{i \in Q_{0}: V_{i} \neq 0\right\}$. Thus for quivers of the form $Q_{\epsilon}$, any indecomposable representation $X_{i, j}^{\epsilon} \in \operatorname{ind}\left(\operatorname{rep}_{\mathbb{k}}\left(Q_{\epsilon}\right)\right)$ has $\operatorname{supp}\left(X_{i, j}^{\epsilon}\right)=[i+1, j]$.

Lemma 25. Let $X_{k, \ell}^{\epsilon}, X_{i, j}^{\epsilon} \in \operatorname{ind}\left(\operatorname{rep}_{\mathbb{R}}\left(Q_{\epsilon}\right)\right)$ and $A:=\left\{a \in\left(Q_{\epsilon}\right)_{1}: s(a), t(a) \in \operatorname{supp}\left(X_{k, \ell}^{\epsilon}\right) \cap\right.$ $\left.\operatorname{supp}\left(X_{i, j}^{\epsilon}\right)\right\}$. Then $\left\langle\underline{\operatorname{dim}}\left(X_{k, \ell}^{\epsilon}\right), \underline{\operatorname{dim}}\left(X_{i, j}^{\epsilon}\right)\right\rangle$ is equal to

$$
\chi_{\operatorname{supp}\left(X_{k, \ell}^{\epsilon}\right) \cap \operatorname{supp}\left(X_{i, j}^{\epsilon}\right)}-\#\left(\left\{a \in\left(Q_{\epsilon}\right)_{1}: s(a) \in \operatorname{supp}\left(X_{k, \ell}^{\epsilon}\right), t(a) \in \operatorname{supp}\left(X_{i, j}^{\epsilon}\right)\right\} \backslash A\right)
$$

where $\chi_{\operatorname{supp}\left(X_{k, \ell}^{\epsilon}\right) \cap \operatorname{supp}\left(X_{i, j}^{\epsilon}\right)}=1$ if $\operatorname{supp}\left(X_{k, \ell}^{\epsilon}\right) \cap \operatorname{supp}\left(X_{i, j}^{\epsilon}\right) \neq \emptyset$ and 0 otherwise.

Proof. We have that

$$
\begin{aligned}
\left\langle\underline{\operatorname{dim}}\left(X_{k, \ell}^{\epsilon}\right), \underline{\operatorname{dim}}\left(X_{i, j}^{\epsilon}\right)\right\rangle= & \sum_{m} \underline{\operatorname{dim}}\left(X_{k, \ell}^{\epsilon}\right)_{m} \underline{\operatorname{dim}}\left(X_{i, j}^{\epsilon}\right)_{m}-\sum_{a} \underline{\operatorname{dim}}\left(X_{k, \ell}^{\epsilon}\right)_{s(a) \underline{\operatorname{dim}}}\left(X_{i, j}^{\epsilon}\right)_{t(a)} \\
= & \#\left(\operatorname{supp}\left(X_{k, \ell}^{\epsilon}\right) \cap \operatorname{supp}\left(X_{i, j}^{\epsilon}\right)\right) \\
& -\#\left\{\alpha \in\left(Q_{\epsilon}\right)_{1}: s(a) \in \operatorname{supp}\left(X_{k, \ell}^{\epsilon}\right), t(a) \in \operatorname{supp}\left(X_{i, j}^{\epsilon}\right)\right\} \\
= & \#\left(\operatorname{supp}\left(X_{k, \ell}^{\epsilon}\right) \cap \operatorname{supp}\left(X_{i, j}^{\epsilon}\right)\right)-\# A \\
& -\#\left(\left\{a \in\left(Q_{\epsilon}\right)_{1}: s(a) \in \operatorname{supp}\left(X_{k, \ell}^{\epsilon}\right), t(a) \in \operatorname{supp}\left(X_{i, j}^{\epsilon}\right)\right\} \backslash A\right) .
\end{aligned}
$$

Observe that if $\operatorname{supp}\left(X_{k, \ell}^{\epsilon}\right) \cap \operatorname{supp}\left(X_{i, j}^{\epsilon}\right) \neq \emptyset$, then $\# A=\#\left(\operatorname{supp}\left(X_{k, \ell}^{\epsilon}\right) \cap \operatorname{supp}\left(X_{i, j}^{\epsilon}\right)\right)-1$. Otherwise $\# A=0$. Thus $\left\langle\underline{\operatorname{dim}}\left(X_{k, \ell}^{\epsilon}\right), \underline{\operatorname{dim}}\left(X_{i, j}^{\epsilon}\right)\right\rangle$ is equal to

$$
\chi_{\operatorname{supp}\left(X_{k, \ell}^{\epsilon}\right) \cap \operatorname{supp}\left(X_{i, j}^{\epsilon}\right)}-\#\left(\left\{a \in\left(Q_{\epsilon}\right)_{1}: s(a) \in \operatorname{supp}\left(X_{k, \ell}^{\epsilon}\right), t(a) \in \operatorname{supp}\left(X_{i, j}^{\epsilon}\right)\right\} \backslash A\right) .
$$

Before presenting the lemmas that we will use in the proof of Lemma 11, we provide a conceptual formulation of their content, which describes when $\operatorname{Hom}_{\mathbb{k} Q_{\epsilon}}(-,-)$ and $\operatorname{Ext}_{\mathbb{k} Q_{\epsilon}}^{1}(-,-)$ vanish for a given type $\mathbb{A}_{n}$ quiver $Q_{\epsilon}$.

Let $X_{k, \ell}^{\epsilon}, X_{i, j}^{\epsilon} \in \operatorname{ind}\left(\operatorname{rep}_{\mathrm{k}}\left(Q_{\epsilon}\right)\right)$ and let $S=\operatorname{supp}\left(X_{k, \ell}^{\epsilon}\right) \cap \operatorname{supp}\left(X_{i, j}^{\epsilon}\right)$. Then we have that $\operatorname{Hom}_{\mathbb{k} Q_{\epsilon}}\left(X_{i, j}^{\epsilon}, X_{k, \ell}^{\epsilon}\right) \neq 0$ if and only if

- $S \neq \emptyset$,

- any $a \in\left(Q_{\epsilon}\right)_{1}$ connecting a vertex $p \in \operatorname{supp}\left(X_{i, j}^{\epsilon}\right) \backslash S$ and a vertex $q \in S$ has $s(a)=q$ and $t(a)=p$, and

- any $a \in\left(Q_{\epsilon}\right)_{1}$ connecting a vertex $p \in \operatorname{supp}\left(X_{k, \ell}^{\epsilon}\right) \backslash S$ and a vertex $q \in S$ has $s(a)=p$ and $t(a)=q$.

Similarly, $\operatorname{Ext}_{\mathbb{k} Q_{\epsilon}}^{1}\left(X_{i, j}^{\epsilon}, X_{k, \ell}^{\epsilon}\right) \neq 0$ if and only if one of the following holds: 
- $\operatorname{Hom}_{\mathbb{k} Q_{\epsilon}}\left(X_{k, \ell}^{\epsilon}, X_{i, j}^{\epsilon}\right) \neq 0$ and $S \subsetneq \operatorname{supp}\left(X_{k, \ell}^{\epsilon}\right), \operatorname{supp}\left(X_{i, j}^{\epsilon}\right)$, or

- $S=\emptyset$ but there is an arrow $a \in\left(Q_{\epsilon}\right)_{1}$ with $s(a) \in \operatorname{supp}\left(X_{i, j}^{\epsilon}\right)$ and $t(a) \in \operatorname{supp}\left(X_{k, \ell}^{\epsilon}\right)$.

In the next four lemmas, we translate the conceptual formulation above into explicit characterizations of when $\operatorname{Hom}_{\mathbb{k} Q_{\epsilon}}(-,-)$ and $\operatorname{Ext}_{\mathbb{k} Q_{\epsilon}}^{1}(-,-)$ vanish. This characterization is in terms of inequalities satisfied by the indices describing a pair of indecomposable representations of $Q_{\epsilon}$ and the entries of $\epsilon$. We will use these explicit lemmas to prove Lemma 11. Since their proofs all use very similar techniques, we only prove Lemma 26.

Lemma 26. Let $X_{k, \ell}^{\epsilon}, X_{i, j}^{\epsilon} \in \operatorname{ind}\left(\operatorname{rep}_{\mathbb{k}}\left(Q_{\epsilon}\right)\right)$. Assume $0 \leqslant i<k<j<\ell \leqslant n$.

i) $\operatorname{Hom}_{\mathbb{k} Q_{\epsilon}}\left(X_{i, j}^{\epsilon}, X_{k, \ell}^{\epsilon}\right) \neq 0$ if and only if $\epsilon_{k}=-$ and $\epsilon_{j}=-$.

ii) $\operatorname{Hom}_{\mathbb{k} Q_{\epsilon}}\left(X_{k, \ell}^{\epsilon}, X_{i, j}^{\epsilon}\right) \neq 0$ if and only if $\epsilon_{k}=+$ and $\epsilon_{j}=+$.

iii) $\operatorname{Ext}_{\mathbb{k} Q_{\epsilon}}^{1}\left(X_{i, j}^{\epsilon}, X_{k, \ell}^{\epsilon}\right) \neq 0$ if and only if $\epsilon_{k}=+$ and $\epsilon_{j}=+$.

iv) $\operatorname{Ext}_{\mathbb{k} Q_{\epsilon}}^{1}\left(X_{k, \ell}^{\epsilon}, X_{i, j}^{\epsilon}\right) \neq 0$ if and only if $\epsilon_{k}=-$ and $\epsilon_{j}=-$.

Proof. We only prove $i$ ) and $i v$ ) as the proof of $i i$ ) is very similar to that of $i$ ), and the proof of $i i i)$ is very similar to that of $i v$ ). To prove $i$ ), first assume there is a nonzero morphism $\theta: X_{i, j}^{\epsilon} \rightarrow X_{k, \ell}^{\epsilon}$. Clearly, $\theta_{s}=0$ if $s \notin[k+1, j]$. If $\theta_{s} \neq 0$ for some $s \in[n]$, then $\theta_{s}=\lambda \cdot \mathrm{id}_{\mathbb{k}}$ for some nonzero $\lambda \in \mathbb{k}$ (i.e., $\theta_{s}$ is a nonzero scalar transformation). As $\theta$ is a morphism of representations, for any $a \in\left(Q_{\epsilon}\right)_{1}$ the equality $\theta_{t(a)} \varphi_{a}^{i, j}=\varphi_{a}^{k, \ell} \theta_{s(a)}$ holds. Thus for any $a \in\left\{a_{k+1}^{\epsilon_{k+1}}, \ldots, a_{j-1}^{\epsilon_{j-1}}\right\}$, we have $\theta_{t(a)}=\theta_{s(a)}$. As $\theta$ is nonzero, this implies that $\theta_{s}=\lambda \cdot \operatorname{id}_{\mathbb{k}}$ for any $s \in[k+1, j]$. If $a=a_{k}^{\epsilon_{k}}$, then we have

$$
\begin{aligned}
\theta_{t(a)} \varphi_{a}^{i, j} & =\varphi_{a}^{k, \ell} \theta_{s(a)} \\
\theta_{t(a)} & =0 .
\end{aligned}
$$

Thus $\epsilon_{k}=-$. Similarly, $\epsilon_{j}=-$.

Conversely, it is easy to see that if $\epsilon_{k}=\epsilon_{j}=-$, then $\theta: X_{i, j}^{\epsilon} \rightarrow X_{k, \ell}^{\epsilon}$ defined by $\theta_{s}=0$ if $s \notin[k+1, j]$ and $\theta_{s}=\mathrm{id}_{\mathbb{k}}$ otherwise is a nonzero morphism.

Next, we prove $i v)$. Observe that by Lemma 25 we have

$$
\begin{aligned}
& \operatorname{dim} \operatorname{Ext}_{\mathbb{k} Q_{\epsilon}}^{1}\left(X_{k, \ell}^{\epsilon}, X_{i, j}^{\epsilon}\right)=\operatorname{dim}_{\operatorname{Hom}_{\mathbb{k} Q_{\epsilon}}}\left(X_{k, \ell}^{\epsilon}, X_{i, j}^{\epsilon}\right)-\left\langle\underline{\operatorname{dim}}\left(X_{k, \ell}^{\epsilon}\right), \underline{\operatorname{dim}}\left(X_{i, j}^{\epsilon}\right)\right\rangle \\
& =\operatorname{dim} \operatorname{Hom}_{\mathbb{k} Q_{\epsilon}}\left(X_{k, \ell}^{\epsilon}, X_{i, j}^{\epsilon}\right)-1 \\
& +\#\left(\left\{b \in\left(Q_{\epsilon}\right)_{1}: s(b) \in \operatorname{supp}\left(X_{k, \ell}^{\epsilon}\right), t(b) \in \operatorname{supp}\left(X_{i, j}^{\epsilon}\right)\right\} \backslash A\right) .
\end{aligned}
$$

Note that \# $\left(\left\{b \in\left(Q_{\epsilon}\right)_{1}: s(b) \in \operatorname{supp}\left(X_{k, \ell}^{\epsilon}\right), t(b) \in \operatorname{supp}\left(X_{i, j}^{\epsilon}\right)\right\} \backslash A\right) \leqslant 2$ with equality if and only if $\epsilon_{k}=\epsilon_{j}=-$.

Suppose $\epsilon_{k}=\epsilon_{j}=-$. By $\left.i\right)$, we have $\operatorname{Hom}_{\mathbb{k} Q_{\epsilon}}\left(X_{i, j}^{\epsilon}, X_{k, \ell}^{\epsilon}\right) \neq 0$ so $\operatorname{Hom}_{\mathbb{k} Q_{\epsilon}}\left(X_{k, \ell}^{\epsilon}, X_{i, j}^{\epsilon}\right)=0$. This means

$$
\begin{aligned}
\operatorname{dim} \operatorname{Ext}_{\mathbb{k} Q_{\epsilon}}^{1}\left(X_{k, \ell}^{\epsilon}, X_{i, j}^{\epsilon}\right) & =\#\left(\left\{b \in\left(Q_{\epsilon}\right)_{1}: s(b) \in \operatorname{supp}\left(X_{k, \ell}^{\epsilon}\right), t(b) \in \operatorname{supp}\left(X_{i, j}^{\epsilon}\right)\right\} \backslash A\right)-1 \\
& =1 .
\end{aligned}
$$

Conversely, suppose $\operatorname{Ext}_{\mathbb{k} Q_{\epsilon}}^{1}\left(X_{k, \ell}^{\epsilon}, X_{i, j}^{\epsilon}\right) \neq 0$. Thus, one checks that there is a nonsplit extension

$$
0 \longrightarrow X_{i, j}^{\epsilon} \stackrel{f}{\longrightarrow} X_{i, \ell}^{\epsilon} \oplus X_{k, j}^{\epsilon} \stackrel{g}{\longrightarrow} X_{k, \ell}^{\epsilon} \longrightarrow 0 .
$$


By Remark 4, this implies that $\operatorname{Hom}_{\mathbb{k} Q_{\epsilon}}\left(X_{k, \ell}^{\epsilon}, X_{i, j}^{\epsilon}\right)=0$, since the composition $h: X_{i, j}^{\epsilon} \stackrel{f_{1}}{\rightarrow}$ $X_{i, \ell}^{\epsilon} \stackrel{g_{1}}{\rightarrow} X_{k, \ell}^{\epsilon}$ is nonzero. Using again that $\operatorname{dimExt}_{\mathbb{k} Q_{\epsilon}}\left(X_{k, \ell}^{\epsilon}, X_{i, j}^{\epsilon}\right) \neq 0$, the formula above for its dimension tells us that $\epsilon_{k}=\epsilon_{j}=-$.

Lemma 27. Let $X_{k, \ell}^{\epsilon}, X_{i, j}^{\epsilon} \in \operatorname{ind}\left(\operatorname{rep}_{\mathbb{k}}\left(Q_{\epsilon}\right)\right)$. Assume $0 \leqslant i<k<\ell<j \leqslant n$.

i) $\operatorname{Hom}_{\mathbb{k} Q_{\epsilon}}\left(X_{i, j}^{\epsilon}, X_{k, \ell}^{\epsilon}\right) \neq 0$ if and only if $\epsilon_{k}=-$ and $\epsilon_{\ell}=+$.

ii) $\operatorname{Hom}_{\mathbb{k} Q_{\epsilon}}\left(X_{k, \ell}^{\epsilon}, X_{i, j}^{\epsilon}\right) \neq 0$ if and only if $\epsilon_{k}=+$ and $\epsilon_{\ell}=-$.

iii) $\operatorname{Ext}_{\mathbb{k} Q_{\epsilon}}^{1}\left(X_{i, j}^{\epsilon}, X_{k, \ell}^{\epsilon}\right) \neq 0$ if and only if $\epsilon_{k}=+$ and $\epsilon_{\ell}=-$.

iv) $\operatorname{Ext}_{\mathbb{k} Q_{\epsilon}}^{1}\left(X_{k, \ell}^{\epsilon}, X_{i, j}^{\epsilon}\right) \neq 0$ if and only if $\epsilon_{k}=-$ and $\epsilon_{\ell}=+$.

Lemma 28. Assume $0 \leqslant i<k<j \leqslant n$. Then

i) $\operatorname{Hom}_{\mathbb{k} Q_{\epsilon}}\left(X_{i, k}^{\epsilon}, X_{k, j}^{\epsilon}\right)=0$ and $\operatorname{Hom}_{\mathbb{k} Q_{\epsilon}}\left(X_{k, j}^{\epsilon}, X_{i, k}^{\epsilon}\right)=0$.

ii) $\operatorname{Ext}_{\mathbb{k} Q_{\epsilon}}^{1}\left(X_{i, k}^{\epsilon}, X_{k, j}^{\epsilon}\right) \neq 0$ if and only if $\epsilon_{k}=+$.

iii) $\operatorname{Ext}_{\mathbb{k} Q_{\epsilon}}^{1}\left(X_{k, j}^{\epsilon}, X_{i, k}^{\epsilon}\right) \neq 0$ if and only if $\epsilon_{k}=-$.

iv) $\operatorname{Hom}_{\mathbb{k} Q_{\epsilon}}\left(X_{i, k}^{\epsilon}, X_{i, j}^{\epsilon}\right) \neq 0$ if and only if $\epsilon_{k}=-$.

v) $\operatorname{Hom}_{\mathbb{k} Q_{\epsilon}}\left(X_{i, j}^{\epsilon}, X_{i, k}^{\epsilon}\right) \neq 0$ if and only if $\epsilon_{k}=+$.

vi) $\operatorname{Ext}_{\mathbb{k} Q_{\epsilon}}^{1}\left(X_{i, k}^{\epsilon}, X_{i, j}^{\epsilon}\right)=0$ and $\operatorname{Ext}_{\mathbb{k} Q_{\epsilon}}^{1}\left(X_{i, j}^{\epsilon}, X_{i, k}^{\epsilon}\right)=0$.

vii) $\operatorname{Hom}_{\mathbb{k} Q_{\epsilon}}\left(X_{k, j}^{\epsilon}, X_{i, j}^{\epsilon}\right) \neq 0$ if and only if $\epsilon_{k}=+$.

viii) $\operatorname{Hom}_{\mathbb{k} Q_{\epsilon}}\left(X_{i, j}^{\epsilon}, X_{k, j}^{\epsilon}\right) \neq 0$ if and only if $\epsilon_{k}=-$.

ix) $\operatorname{Ext}_{\mathbb{k} Q_{\epsilon}}^{1}\left(X_{k, j}^{\epsilon}, X_{i, j}^{\epsilon}\right)=0$ and $\operatorname{Ext}_{\mathbb{k} Q_{\epsilon}}^{1}\left(X_{i, j}^{\epsilon}, X_{k, j}^{\epsilon}\right)=0$.

Lemma 29. Let $X_{k, \ell}^{\epsilon}, X_{i, j}^{\epsilon} \in \operatorname{ind}\left(\operatorname{rep}_{\mathbb{k}}\left(Q_{\epsilon}\right)\right)$. Assume $0 \leqslant i<j<k<\ell \leqslant n$. Then

i) $\operatorname{Hom}_{\mathbb{k} Q_{\epsilon}}\left(X_{i, j}^{\epsilon}, X_{k, \ell}^{\epsilon}\right)=0, \operatorname{Hom}_{\mathbb{k} Q_{\epsilon}}\left(X_{k, \ell}^{\epsilon}, X_{i, j}^{\epsilon}\right)=0$,

ii) $\operatorname{Ext}_{\mathbb{k} Q_{\epsilon}}^{1}\left(X_{i, j}^{\epsilon}, X_{k, \ell}^{\epsilon}\right)=0, \operatorname{Ext}_{\mathbb{k} Q_{\epsilon}}^{1}\left(X_{k, \ell}^{\epsilon}, X_{i, j}^{\epsilon}\right)=0$.

We begin our proof of Lemma 11 by giving a formula for a good choice of representative monotone curves $\gamma_{\epsilon}(i, j) \in c(i, j)$ for all strands $c(i, j)$. Let $\varphi: \mathbb{R} \rightarrow \mathbb{R}$ be any fixed smooth $\left(C^{1}\right)$ function satisfying the equation $\varphi\left(x_{k}\right)=\epsilon_{k} \sqrt{x_{k}}$ for all $\left(x_{k}, 0\right) \in \mathcal{S}_{n, \epsilon}$. For $0 \leqslant i<j \leqslant n$ let $f_{i j}:\left[x_{i}, x_{j}\right] \rightarrow \mathbb{R}$ be the smooth function:

$$
f_{i j}(t)=\epsilon_{i} \sqrt{x_{i}} \frac{x_{j}-t}{x_{j}-x_{i}}+\epsilon_{j} \sqrt{x_{j}} \frac{t-x_{i}}{x_{j}-x_{i}}-\varphi(t)
$$

Note that the graph of the function $f_{i j}+\varphi$ is a straight line from $\left(x_{i}, \epsilon_{i} \sqrt{x_{i}}\right)$ to $\left(x_{j}, \epsilon_{j} \sqrt{x_{j}}\right)$.

Lemma 30. The graph of the function $f_{i j}:\left[x_{i}, x_{j}\right] \rightarrow \mathbb{R}$ is a monotone curve $\gamma_{\epsilon}(i, j) \in$ $c(i, j)$.

Proof. We follow Remark 8. For $t=x_{i}, f\left(x_{i}\right)=\epsilon_{i} \sqrt{x_{i}}-\epsilon_{i} \sqrt{x_{i}}=0$. Similarly, $f\left(x_{j}\right)=0$. Now, let $i<k<j$. Since the function $y=\sqrt{x}$ is concave down, the weighted average of $\sqrt{x_{i}}, \sqrt{x_{j}}$ will be strictly smaller than $\sqrt{x_{k}}$ :

$$
\left|f_{i j}\left(x_{k}\right)+\varphi\left(x_{k}\right)\right|<\sqrt{x_{k}} .
$$

Since $\left|\varphi\left(x_{k}\right)\right|=\sqrt{x_{k}}$, the sign of $f_{i j}\left(x_{k}\right)$ must be $-\epsilon_{k}$ as required by Remark 8 . 
Lemma 31. Let $c\left(i_{1}, j_{1}\right)$ and $c\left(i_{2}, j_{2}\right)$ be distinct strands on $\mathcal{S}_{n, \epsilon}$ that intersect nontrivially. Then $c\left(i_{1}, j_{1}\right)$ and $c\left(i_{2}, j_{2}\right)$ do not share an endpoint.

Proof. Suppose $c\left(i_{1}, j_{1}\right)$ and $c\left(i_{2}, j_{2}\right)$ share an endpoint. Then the linear functions $f_{i_{1} j_{1}}+\varphi$ and $f_{i_{2} j_{2}}+\varphi$ agree at one endpoint and therefore must be disjoint at all other points. So, $f_{i_{1} j_{1}} \neq f_{i_{2} j_{2}}$ except at an endpoint and $c\left(i_{1}, j_{1}\right), c\left(i_{2}, j_{2}\right)$ cannot intersect nontrivially.

Lemma 32. Two distinct strands $c\left(i_{1}, j_{1}\right)$ and $c\left(i_{2}, j_{2}\right)$ on $\mathcal{S}_{n, \epsilon}$ intersect nontrivially if and only if the representative monotone curves $\gamma_{\epsilon}\left(i_{1}, j_{1}\right)$ and $\gamma_{\epsilon}\left(i_{2}, j_{2}\right)$ have a unique crossing.

Proof. By the previous lemma we may assume that $c\left(i_{1}, j_{1}\right)$ and $c\left(i_{2}, j_{2}\right)$ do not share an endpoint.

If $\gamma_{\epsilon}\left(i_{1}, j_{1}\right)$ and $\gamma_{\epsilon}\left(i_{2}, j_{2}\right)$ do not intersect then, by definition, $c\left(i_{1}, j_{1}\right)$ and $c\left(i_{2}, j_{2}\right)$ do not intersect nontrivially. So, we may assume that $\gamma_{\epsilon}\left(i_{1}, j_{1}\right)$ and $\gamma_{\epsilon}\left(i_{2}, j_{2}\right)$ intersect. Then the linear functions $f_{i_{1} j_{1}}+\varphi, f_{i_{2} j_{2}}+\varphi$ agree at one point. The slopes of these lines must be different, otherwise one curve $\gamma_{\epsilon}\left(i_{1}, j_{1}\right)$ or $\gamma_{\epsilon}\left(i_{2}, j_{2}\right)$ would contain an endpoint of the other. Therefore, $\gamma_{\epsilon}\left(i_{1}, j_{1}\right)$ and $\gamma_{\epsilon}\left(i_{2}, j_{2}\right)$ intersect transversely at one point. It remains to show that $c\left(i_{1}, j_{1}\right)$ and $c\left(i_{2}, j_{2}\right)$ intersect nontrivially, i.e., that any other pair of representatives will intersect in their interiors.

Assuming that one pair of transversely intersecting representative curves $\gamma_{k} \in c\left(i_{k}, j_{k}\right)$ with $k \in[2]$ meets an odd number of times, we will show that all of them do. This will prove the lemma. Without loss of generality, we assume $i_{1}<i_{2}$. Since $c\left(i_{1}, j_{1}\right)$ and $c\left(i_{2}, j_{2}\right)$ do not share a common endpoint there are two cases:

a) $i_{1}<i_{2}<j_{1}<j_{2}$

b) $i_{1}<i_{2}<j_{2}<j_{1}$.

Suppose that case $a$ ) holds. If $\epsilon_{i_{2}}=-$ (resp., $\epsilon_{i_{2}}=+$ ), then $\gamma_{1}$ passes over (resp., underneath) $\gamma_{2}$ at its left endpoint $\left(x_{i_{2}}, 0\right)$. Since $\gamma_{1}, \gamma_{2}$ cross an odd number of times, the right hand endpoint of $\gamma_{1}$ must lie under (resp., over) $\gamma_{2}$, i.e., $\epsilon_{j_{1}}=-$ (resp., $\epsilon_{j_{1}}=+$ ). In both subcases, $\epsilon_{i_{2}}=\epsilon_{j_{1}}$ and $\gamma_{1}$ and $\gamma_{2}$ must switch vertical order between $\left(x_{i_{2}}, 0\right)$ and $\left(x_{j_{1}}, 0\right)$. So, all choices of $\gamma_{1}, \gamma_{2}$ must cross an odd number of times if the crossings are transverse.

The proof in case $b$ ) is very similar. Again the monotone curves $\gamma_{1}, \gamma_{2}$ must switch their vertical order between $\left(x_{i_{2}}, 0\right)$ and $\left(x_{j_{1}}, 0\right)$ which, in case b) means that $\epsilon_{i_{2}} \neq \epsilon_{j_{2}}$. Then all choices of $\gamma_{1}, \gamma_{2}$ will intersect an odd number of times if the intersections are transverse.

Remark 33. If $c\left(i_{1}, j_{1}\right)$ and $c\left(i_{2}, j_{2}\right)$ are two distinct strands on $\mathcal{S}_{n, \epsilon}$ that do not intersect nontrivially, then the representative monotone curves $\gamma_{\epsilon}\left(i_{1}, j_{1}\right)$ and $\gamma_{\epsilon}\left(i_{2}, j_{2}\right)$ are nonintersecting, except possibly at their endpoints.

We now arrive at the proof of Lemma 11. The proofs of a), b), and c) are a case-bycase analysis where the cases are given in terms of the entries of $\epsilon$ and the inequalities satisfied by the indices describing a pair of indecomposable representations of $Q_{\epsilon}$. The representatives $\gamma_{\epsilon}(U)$ of $U=X_{i, j}^{\epsilon}$ claimed by Lemma 11 are given by $\gamma_{\epsilon}(U)=\gamma_{\epsilon}(i, j)$ which have the required properties by Lemma 32 and Remark 33 above. 
Proof of Lemma 11 a). Let $X_{i, j}^{\epsilon}=U$ and $X_{k, \ell}^{\epsilon}=V$. Assume that the strands $\Phi_{\epsilon}\left(X_{i, j}^{\epsilon}\right)$ and $\Phi_{\epsilon}\left(X_{k, \ell}^{\epsilon}\right)$ intersect nontrivially. By Lemma 31, we can assume without loss of generality that either $0 \leqslant i<k<j<\ell \leqslant n$ or $0 \leqslant i<k<\ell<j \leqslant n$. By Lemma 32, we can represent $\Phi_{\epsilon}\left(X_{i, j}^{\epsilon}\right)$ and $\Phi_{\epsilon}\left(X_{k, \ell}^{\epsilon}\right)$ by monotone curves $\gamma_{i, j}$ and $\gamma_{k, \ell}$ that have a unique crossing. Furthermore, we can assume that this unique crossing occurs between $\left(x_{k}, 0\right)$ and $\left(x_{k+1}, 0\right)$. There are four possible cases:
i) $\epsilon_{k}=\epsilon_{k+1}=-$,
ii) $\epsilon_{k}=-$ and $\epsilon_{k+1}=+$,
iii) $\epsilon_{k}=\epsilon_{k+1}=+$,
iv) $\epsilon_{k}=+$ and $\epsilon_{k+1}=-$.

We illustrate these cases up to isotopy in Figure 4. We see that in cases $i$ ) and $i i$ ) (resp., iii) and $i v)) \gamma_{k, \ell}$ lies

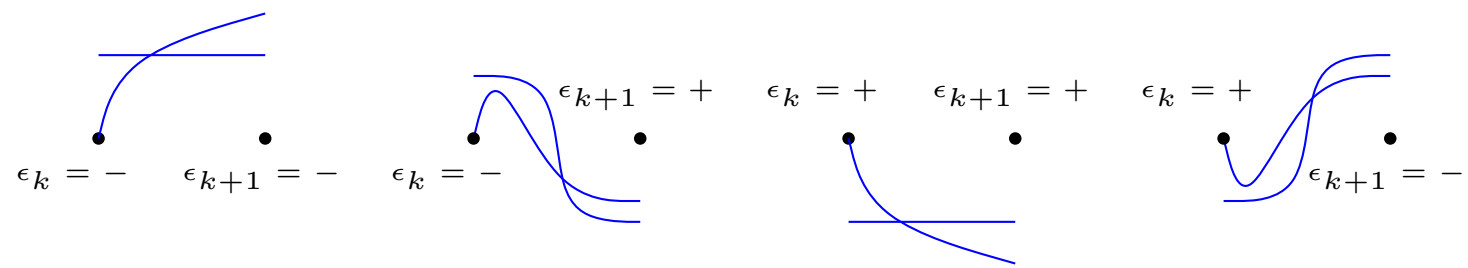

Figure 4: The four types of crossings.

above (resp., below) $\gamma_{i, j}$ inside of $\left\{(x, y) \in \mathbb{R}^{2}: x_{k+1} \leqslant x \leqslant x_{\min \{\ell, j\}}\right\}$.

Suppose $\gamma_{k, \ell}$ lies above $\gamma_{i, j}$ inside $\left\{(x, y) \in \mathbb{R}^{2}: x_{k+1} \leqslant x \leqslant x_{\min \{\ell, j\}}\right\}$. Then

$$
\epsilon_{\min \{\ell, j\}}=\left\{\begin{array}{l}
+: \min \{\ell, j\}=\ell \\
-\quad: \min \{\ell, j\}=j
\end{array}\right.
$$

otherwise $\gamma_{k, \ell}$ and $\gamma_{i, j}$ would have a nonunique crossing. If $\min \{\ell, j\}=\ell$, we have $0 \leqslant i<$ $k<\ell<j \leqslant n, \epsilon_{k}=-$, and $\epsilon_{\ell}=+$. Now by Lemma 27, we have that $\operatorname{Hom}_{\mathbb{k} Q_{\epsilon}}\left(X_{i, j}^{\epsilon}, X_{k, \ell}^{\epsilon}\right) \neq$ 0 and $\operatorname{Ext}_{\mathbb{k} Q_{\epsilon}}^{1}\left(X_{k, \ell}^{\epsilon}, X_{i, j}^{\epsilon}\right) \neq 0$. If $\min \{\ell, j\}=j$, then $0 \leqslant i<k<j<\ell \leqslant n, \epsilon_{k}=-$, and $\epsilon_{j}=-$. Thus, by Lemma 26, we have that $\operatorname{Hom}_{\mathbb{k} Q_{\epsilon}}\left(X_{i, j}^{\epsilon}, X_{k, \ell}^{\epsilon}\right) \neq 0$ and $\operatorname{Ext}_{\mathbb{k} Q_{\epsilon}}^{1}\left(X_{k, \ell}^{\epsilon}, X_{i, j}^{\epsilon}\right) \neq$ 0 .

Similarly, if $\gamma_{i, j}$ lies above $\gamma_{k, \ell}$ inside $\left\{(x, y) \in \mathbb{R}^{2}: x_{k+1} \leqslant x \leqslant x_{\min \{\ell, j\}}\right\}$, it follows that

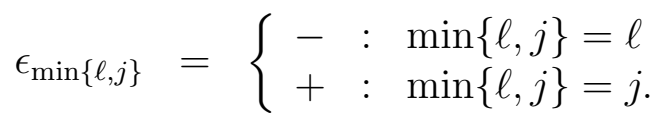

If $\min \{\ell, j\}=\ell$, then $\operatorname{Hom}_{\mathbb{k} Q_{\epsilon}}\left(X_{k, \ell}^{\epsilon}, X_{i, j}^{\epsilon}\right) \neq 0$ and $\operatorname{Ext}_{\mathbb{k} Q_{\epsilon}}^{1}\left(X_{i, j}^{\epsilon}, X_{k, \ell}^{\epsilon}\right) \neq 0$ via Lemma 27. If $\min \{\ell, j\}=j$, then Lemma 26 implies $\operatorname{Hom}_{\mathbb{k} Q_{\epsilon}}\left(X_{k, \ell}^{\epsilon}, X_{i, j}^{\epsilon}\right) \neq 0$ and $\operatorname{Ext}_{\mathbb{k} Q_{\epsilon}}^{1}\left(X_{i, j}^{\epsilon}, X_{k, \ell}^{\epsilon}\right) \neq 0$. Thus we conclude that neither $\left(X_{i, j}^{\epsilon}, X_{k, \ell}^{\epsilon}\right)$ nor $\left(X_{k, \ell}^{\epsilon}, X_{i, j}^{\epsilon}\right)$ are exceptional pairs.

Conversely, assume that neither $(U, V)$ nor $(V, U)$ are exceptional pairs where $X_{i, j}^{\epsilon}=U$ and $X_{k, \ell}^{\epsilon}=V$. Then at least one of the following is true: 
a) $\operatorname{Hom}_{\mathbb{k} Q_{\epsilon}}\left(X_{i, j}^{\epsilon}, X_{k, \ell}^{\epsilon}\right) \neq 0$ and $\operatorname{Hom}_{\mathbb{k} Q_{\epsilon}}\left(X_{k, \ell}^{\epsilon}, X_{i, j}^{\epsilon}\right) \neq 0$,

b) $\operatorname{Hom}_{\mathbb{k} Q_{\epsilon}}\left(X_{i, j}^{\epsilon}, X_{k, \ell}^{\epsilon}\right) \neq 0$ and $\operatorname{Ext}_{\mathbb{k} Q_{\epsilon}}^{1}\left(X_{k, \ell}^{\epsilon}, X_{i, j}^{\epsilon}\right) \neq 0$,

c) $\operatorname{Ext}_{\mathbb{k} Q_{\epsilon}}^{1}\left(X_{i, j}^{\epsilon}, X_{k, \ell}^{\epsilon}\right) \neq 0$ and $\operatorname{Hom}_{\mathbb{k} Q_{\epsilon}}\left(X_{k, \ell}^{\epsilon}, X_{i, j}^{\epsilon}\right) \neq 0$,

d) $\operatorname{Ext}_{\mathbb{k} Q_{\epsilon}}^{1}\left(X_{i, j}^{\epsilon}, X_{k, \ell}^{\epsilon}\right) \neq 0$ and $\operatorname{Ext}_{\mathbb{k} Q_{\epsilon}}^{1}\left(X_{k, \ell}^{\epsilon}, X_{i, j}^{\epsilon}\right) \neq 0$.

As $X_{i, j}^{\epsilon}$ and $X_{k, \ell}^{\epsilon}$ are indecomposable and nonisomorphic, we have $\operatorname{Hom}_{\mathbb{k} Q_{\epsilon}}\left(X_{i, j}^{\epsilon}, X_{k, \ell}^{\epsilon}\right)=$ 0 or $\operatorname{Hom}_{\mathbb{k} Q_{\epsilon}}\left(X_{k, \ell}^{\epsilon}, X_{i, j}^{\epsilon}\right)=0$ by Remark 4. Without loss of generality, assume that $\operatorname{Hom}_{\mathbb{k} Q_{\epsilon}}\left(X_{k, \ell}^{\epsilon}, X_{i, j}^{\epsilon}\right)=0$. Thus $\left.b\right)$ or $\left.d\right)$ hold so $\operatorname{Ext}_{\mathbb{k} Q_{\epsilon}}^{1}\left(X_{k, \ell}^{\epsilon}, X_{i, j}^{\epsilon}\right) \neq 0$. Then Lemma 28 and Lemma 29 imply that $0 \leqslant i<k<j<\ell \leqslant n$ or $0 \leqslant i<k<\ell<j \leqslant n$.

If $0 \leqslant i<k<j<\ell<n$, we have $\epsilon_{k}=\epsilon_{j}=-$ by Lemma 26 as $\operatorname{Hom}_{\mathbb{k} Q_{\epsilon}}\left(X_{i, j}^{\epsilon}, X_{k, \ell}^{\epsilon}\right) \neq 0$ and $\operatorname{Ext}_{\mathbb{k} Q_{\epsilon}}^{1}\left(X_{k, \ell}^{\epsilon}, X_{i, j}^{\epsilon}\right) \neq 0$. Let $\gamma_{i, j} \in \Phi_{\epsilon}\left(X_{i, j}^{\epsilon}\right)$ and $\gamma_{k, \ell} \in \Phi_{\epsilon}\left(X_{k, \ell}^{\epsilon}\right)$. We can assume that there exists $\delta(k)>0$ such that $\gamma_{i, j}$ and $\gamma_{k, \ell}$ have no intersection inside $\left\{(x, y) \in \mathbb{R}^{2}\right.$ : $\left.x_{k} \leqslant x \leqslant x_{k}+\delta(k)\right\}$. This implies that $\gamma_{i, j}$ lies above $\gamma_{k, \ell}$ inside $\left\{(x, y) \in \mathbb{R}^{2}: x_{k} \leqslant x \leqslant\right.$ $\left.x_{k}+\delta(k)\right\}$. Similarly, we can assume there exists $\delta(j)>0$ such that $\gamma_{i, j}$ and $\gamma_{k, \ell}$ have no intersection inside $\left\{(x, y) \in \mathbb{R}^{2}: x_{j}-\delta(j) \leqslant x \leqslant x_{j}\right\}$. Thus $\gamma_{i, j}$ lies below $\gamma_{k, \ell}$ inside $\left\{(x, y) \in \mathbb{R}^{2}: x_{j}-\delta(j) \leqslant x \leqslant x_{j}\right\}$. This means $\gamma_{i, j}$ and $\gamma_{k, \ell}$ must have at least one point of intersection. Thus $\Phi_{\epsilon}\left(X_{i, j}^{\epsilon}\right)$ and $\Phi_{\epsilon}\left(X_{k, \ell}^{\epsilon}\right)$ intersect nontrivially. An analogous argument shows that if $0 \leqslant i<k<\ell<j \leqslant n$, then $\Phi_{\epsilon}\left(X_{i, j}^{\epsilon}\right)$ and $\Phi_{\epsilon}\left(X_{k, \ell}^{\epsilon}\right)$ intersect nontrivially.

Proof of Lemma 11 b). Assume that $\Phi_{\epsilon}(U)$ is clockwise from $\Phi_{\epsilon}(V)$. Then we have that one of the following holds:

a) $X_{k, j}^{\epsilon}=U$ and $X_{i, k}^{\epsilon}=V$ for some $0 \leqslant i<k<j \leqslant n$,

b) $X_{i, k}^{\epsilon}=U$ and $X_{k, j}^{\epsilon}=V$ for some $0 \leqslant i<k<j \leqslant n$,

c) $X_{i, j}^{\epsilon}=U$ and $X_{i, k}^{\epsilon}=V$ for some $0 \leqslant i<j \leqslant n$ and $0 \leqslant i<k \leqslant n$,

d) $X_{i, j}^{\epsilon}=U$ and $X_{k, j}^{\epsilon}=V$ for some $0 \leqslant i<j \leqslant n$ and $0 \leqslant k<j \leqslant n$.

In Case $a$ ), we have that $\epsilon_{k}=-$ since $\Phi_{\epsilon}\left(X_{k, j}^{\epsilon}\right)$ is clockwise from $\Phi_{\epsilon}\left(X_{i, k}^{\epsilon}\right)$. By Lemma $28 i$ ) and $i i)$, we have $\operatorname{Hom}_{\mathbb{k} Q_{\epsilon}}\left(X_{i, k}^{\epsilon}, X_{k, j}^{\epsilon}\right)=0$ and $\operatorname{Ext}_{\mathbb{k} Q_{\epsilon}}^{1}\left(X_{i, k}^{\epsilon}, X_{k, j}^{\epsilon}\right)=0$. Thus $\left(X_{k, j}^{\epsilon}, X_{i, k}^{\epsilon}\right)$ is an exceptional pair. By Lemma $\left.28 i i i\right)$, we have that $\operatorname{Ext}_{\mathbb{k} Q_{\epsilon}}^{1}\left(X_{k, j}^{\epsilon}, X_{i, k}^{\epsilon}\right) \neq 0$. Thus $\left(X_{i, k}^{\epsilon}, X_{k, j}^{\epsilon}\right)$ is not an exceptional pair.

In Case b), we have that $\epsilon_{k}=+$ since $\Phi_{\epsilon}\left(X_{i, k}^{\epsilon}\right)$ is clockwise from $\Phi_{\epsilon}\left(X_{k, j}^{\epsilon}\right)$. By Lemma $28 i$ ) and $i i i)$, we have that $\operatorname{Hom}_{\mathbb{k} Q_{\epsilon}}\left(X_{k, j}^{\epsilon}, X_{i, k}^{\epsilon}\right)=0$ and $\operatorname{Ext}_{\mathbb{k} Q_{\epsilon}}^{1}\left(X_{k, j}^{\epsilon}, X_{i, k}^{\epsilon}\right)=0$. Thus $\left(X_{i, k}^{\epsilon}, X_{k, j}^{\epsilon}\right)$ is an exceptional pair. By Lemma $\left.28 i i\right)$, we have $\operatorname{Ext}_{\mathbb{k} Q_{\epsilon}}^{1}\left(X_{i, k}^{\epsilon}, X_{k, j}^{\epsilon}\right) \neq 0$. Thus $\left(X_{k, j}^{\epsilon}, X_{i, k}^{\epsilon}\right)$ is not an exceptional pair.

In Case $c$ ), if $j<k$, it follows that $\epsilon_{j}=-$. Indeed, since $\Phi_{\epsilon}\left(X_{i, j}^{\epsilon}\right)$ and $\Phi_{\epsilon}\left(X_{i, k}^{\epsilon}\right)$ share an endpoint, the two do not intersect nontrivially by Lemma 31. As $\Phi_{\epsilon}\left(X_{i, j}^{\epsilon}\right)$ is clockwise from $\Phi_{\epsilon}\left(X_{i, k}^{\epsilon}\right)$, Remark 33 asserts that we can choose monotone curves $\gamma_{i, k} \in \Phi_{\epsilon}\left(X_{i, k}^{\epsilon}\right)$ and $\gamma_{i, j} \in \Phi_{\epsilon}\left(X_{i, j}^{\epsilon}\right)$ such that $\gamma_{i, k}$ lies strictly above $\gamma_{i, j}$ on $\left\{(x, y) \in \mathbb{R}^{2}: x_{i}<x \leqslant\right.$ $x_{j}$. Thus $\epsilon_{j}=-$. By Lemma $28 v$ ) and $\left.v i\right)$, we have that $\operatorname{Hom}_{\mathbb{k} Q_{\epsilon}}\left(X_{i, k}^{\epsilon}, X_{i, j}^{\epsilon}\right)=0$ and $\operatorname{Ext}_{\mathbb{k} Q_{\epsilon}}^{1}\left(X_{i, k}^{\epsilon}, X_{i, j}^{\epsilon}\right)=0$ so that $\left(X_{i, j}^{\epsilon}, X_{i, k}^{\epsilon}\right)$ is an exceptional pair. By Lemma $28 i v$ ), we have that $\operatorname{Hom}_{\mathbb{k} Q_{\epsilon}}\left(X_{i, j}^{\epsilon}, X_{i, k}^{\epsilon}\right) \neq 0$. Thus $\left(X_{i, k}^{\epsilon}, X_{i, j}^{\epsilon}\right)$ is not an exceptional pair.

Similarly, one shows that if $k<j$, then $\epsilon_{k}=+$. By Lemma $28 i v$ ) and $v i$ ), we have that $\operatorname{Hom}_{\mathbb{k} Q_{\epsilon}}\left(X_{i, k}^{\epsilon}, X_{i, j}^{\epsilon}\right)=0$ and $\operatorname{Ext}_{\mathbb{k} Q_{\epsilon}}^{1}\left(X_{i, k}^{\epsilon}, X_{i, j}^{\epsilon}\right)=0$ so that $\left(X_{i, j}^{\epsilon}, X_{i, k}^{\epsilon}\right)$ is an exceptional pair. By Lemma $28 v$ ), we have that $\operatorname{Hom}_{\mathbb{k} Q_{\epsilon}}\left(X_{i, j}^{\epsilon}, X_{i, k}^{\epsilon}\right) \neq 0$. Thus $\left(X_{i, k}^{\epsilon}, X_{i, j}^{\epsilon}\right)$ is not an exceptional pair. The proof in Case $d$ ) is completely analogous to the proof in Case $c$ ) so 
we omit it.

Conversely, let $U=X_{i, j}^{\epsilon}$ and $V=X_{k, \ell}^{\epsilon}$ and assume that $\left(X_{i, j}^{\epsilon}, X_{k, \ell}^{\epsilon}\right)$ is an exceptional pair and $\left(X_{k, \ell}^{\epsilon}, X_{i, j}^{\epsilon}\right)$ is not an exceptional pair. This implies that at least one of the following holds:

1) $\operatorname{Hom}_{\mathbb{k} Q_{\epsilon}}\left(X_{k, \ell}^{\epsilon}, X_{i, j}^{\epsilon}\right)=0, \operatorname{Ext}_{\mathbb{k} Q_{\epsilon}}^{1}\left(X_{k, \ell}^{\epsilon}, X_{i, j}^{\epsilon}\right)=0$, and $\operatorname{Hom}_{\mathbb{k} Q_{\epsilon}}\left(X_{i, j}^{\epsilon}, X_{k, \ell}^{\epsilon}\right) \neq 0$,

2) $\operatorname{Hom}_{\mathbb{k} Q_{\epsilon}}\left(X_{k, \ell}^{\epsilon}, X_{i, j}^{\epsilon}\right)=0, \operatorname{Ext}_{\mathbb{k} Q_{\epsilon}}^{1}\left(X_{k, \ell}^{\epsilon}, X_{i, j}^{\epsilon}\right)=0$, and $\operatorname{Ext}_{\mathbb{k} Q_{\epsilon}}^{1}\left(X_{i, j}^{\epsilon}, X_{k, \ell}^{\epsilon}\right) \neq 0$.

By Lemma 29, we know that $[i, j] \cap[k, \ell] \neq \emptyset$. This implies that either

i) $\Phi_{\epsilon}\left(X_{i, j}^{\epsilon}\right)$ and $\Phi_{\epsilon}\left(X_{k, \ell}^{\epsilon}\right)$ share an endpoint,

ii) $0 \leqslant i<k<j<\ell \leqslant n$,

iii) $0 \leqslant i<k<\ell<j \leqslant n$,

iv) $0 \leqslant k<i<\ell<j \leqslant n$, or

v) $0 \leqslant k<i<j<\ell \leqslant n$.

We will show that $\Phi_{\epsilon}\left(X_{i, j}^{\epsilon}\right)$ and $\Phi_{\epsilon}\left(X_{k, \ell}^{\epsilon}\right)$ share an endpoint.

Suppose $0 \leqslant i<k<j<\ell \leqslant n$. Since $\operatorname{Hom}_{\mathbb{k} Q_{\epsilon}}\left(X_{k, \ell}^{\epsilon}, X_{i, j}^{\epsilon}\right)=0, \operatorname{Ext}_{\mathbb{k} Q_{\epsilon}}^{1}\left(X_{k, \ell}^{\epsilon}, X_{i, j}^{\epsilon}\right)=0$, we have by Lemma $26 \mathrm{ii}$ ) and $i v$ ) that either $\epsilon_{k}=-$ and $\epsilon_{j}=+$ or $\epsilon_{k}=+$ and $\epsilon_{j}=-$. However, as $\operatorname{Hom}_{\mathbb{k} Q_{\epsilon}}\left(X_{i, j}^{\epsilon}, X_{k, \ell}^{\epsilon}\right) \neq 0$ or $\operatorname{Ext}_{\mathbb{k} Q_{\epsilon}}^{1}\left(X_{i, j}^{\epsilon}, X_{k, \ell}^{\epsilon}\right) \neq 0$, Lemma $26 i$ ) and $\left.i i i\right)$ assert that $\epsilon_{k}=\epsilon_{j}=-$ or $\epsilon_{k}=\epsilon_{j}=+$. This is a contradiction. Thus, $i, j, k, \ell$ do not satisfy $0 \leqslant i<k<j<\ell \leqslant n$, and by a similar argument, they also do not satisfy $0 \leqslant k<i<\ell<j \leqslant n$.

Suppose $0 \leqslant i<k<\ell<j \leqslant n$. Since $\operatorname{Hom}_{\mathbb{k} Q_{\epsilon}}\left(X_{k, \ell}^{\epsilon}, X_{i, j}^{\epsilon}\right)=0, \operatorname{Ext}_{\mathbb{k} Q_{\epsilon}}^{1}\left(X_{k, \ell}^{\epsilon}, X_{i, j}^{\epsilon}\right)=0$, we have by Lemma $27 i i$ ) and $i v$ ) that either $\epsilon_{k}=\epsilon_{\ell}=+$ or $\epsilon_{k}=\epsilon_{\ell}=-$. However, as $\operatorname{Hom}_{\mathbb{k} Q_{\epsilon}}\left(X_{i, j}^{\epsilon}, X_{k, \ell}^{\epsilon}\right) \neq 0$ or $\operatorname{Ext}_{\mathbb{k} Q_{\epsilon}}^{1}\left(X_{i, j}^{\epsilon}, X_{k, \ell}^{\epsilon}\right) \neq 0$, Lemma $\left.27 i\right)$ and $\left.i i i\right)$ we have that $\epsilon_{k}=-$ and $\epsilon_{\ell}=+$ or $\epsilon_{k}=+$ and $\epsilon_{\ell}=-$. This is a contradiction. Thus, $i, j, k, \ell$ do not satisfy $0 \leqslant i<k<\ell<j \leqslant n$, and by an analogous argument, they also do not satisfy $0 \leqslant k<i<j<\ell \leqslant n$.

We conclude that $\Phi_{\epsilon}(U)$ and $\Phi_{\epsilon}(V)$ share an endpoint. Thus we have that one of the following holds where we forget the previous roles played by $i, j, k$ :

a) $X_{k, j}^{\epsilon}=U$ and $X_{i, k}^{\epsilon}=V$ for some $0 \leqslant i<k<j \leqslant n$,

b) $X_{i, k}^{\epsilon}=U$ and $X_{k, j}^{\epsilon}=V$ for some $0 \leqslant i<k<j \leqslant n$,

c) $X_{i, j}^{\epsilon}=U$ and $X_{i, k}^{\epsilon}=V$ for some $0 \leqslant i<j \leqslant n$ and $0 \leqslant i<k \leqslant n$,

d) $X_{i, j}^{\epsilon}=U$ and $X_{k, j}^{\epsilon}=V$ for some $0 \leqslant i<j \leqslant n$ and $0 \leqslant k<j \leqslant n$.

Suppose Case $a$ ) holds. We know that $\operatorname{Ext}_{\mathbb{k} Q_{\epsilon}}^{1}\left(X_{i, k}^{\epsilon}, X_{k, j}^{\epsilon}\right)=0$ since $(U, V)$ is an exceptional pair. By Lemma $28 \mathrm{ii}$ ), we have that $\epsilon_{k}=-$. Thus $\Phi_{\epsilon}(U)$ is clockwise from $\Phi_{\epsilon}(V)$.

Suppose Case $b$ ) holds. We know that $\operatorname{Ext}_{\mathbb{k} Q_{\epsilon}}^{1}\left(X_{k, j}^{\epsilon}, X_{i, k}^{\epsilon}\right)=0$ since $(U, V)$ is an exceptional pair. By Lemma 28 iii), we have that $\epsilon_{k}=+$. Thus $\Phi_{\epsilon}(U)$ is clockwise from $\Phi_{\epsilon}(V)$.

Suppose Case $c$ ) holds. Assume $k<j$. Then Lemma $28 i v$ ) and the fact that $\operatorname{Hom}_{\mathbb{k} Q_{\epsilon}}\left(X_{i, k}^{\epsilon}, X_{i, j}^{\epsilon}\right)=0$ imply that $\epsilon_{k}=+$. Thus we have that $\Phi_{\epsilon}(U)=\Phi_{\epsilon}\left(X_{i, j}^{\epsilon}\right)$ is clockwise from $\Phi_{\epsilon}(V)=\Phi_{\epsilon}\left(X_{i, k}^{\epsilon}\right)$. Now suppose $j<k$. Then Lemma $28 v$ ) and $\operatorname{Hom}_{\mathbb{k} Q_{\epsilon}}\left(X_{i, k}^{\epsilon}, X_{i, j}^{\epsilon}\right)=0$ imply that $\epsilon_{j}=-$. Thus we have that $\Phi_{\epsilon}(U)=\Phi_{\epsilon}\left(X_{i, j}^{\epsilon}\right)$ is clockwise from $\Phi_{\epsilon}(V)=\Phi_{\epsilon}\left(X_{i, k}^{\epsilon}\right)$. The proof in Case $\left.d\right)$ is very similar so we omit it. 
Proof of Lemma $11 \mathrm{c})$. Observe that two strands $c\left(i_{1}, j_{1}\right)$ and $c\left(i_{2}, j_{2}\right)$ share an endpoint if and only if one of the two strands is clockwise from the other. Thus Lemma $11 a$ ) and $b)$ imply that $\Phi_{\epsilon}(U)$ and $\Phi_{\epsilon}(V)$ do not intersect at any of their endpoints and they do not intersect nontrivially if and only if both $(U, V)$ and $(V, U)$ are exceptional pairs.

\section{Mixed cobinary trees}

We recall the definition of an $\epsilon$-mixed cobinary tree and construct a bijection between the set of (isomorphism classes of) such trees and the set of maximal oriented strand diagrams on $\mathcal{S}_{n, \epsilon}$.

Definition 34 ([IO13]). Given a sign function $\epsilon:[0, n] \rightarrow\{+,-\}$, an $\boldsymbol{\epsilon}$-mixed cobinary tree (MCT) is a tree $T$ embedded in $\mathbb{R}^{2}$ with vertex set $\left\{\left(i, y_{i}\right): i \in[0, n]\right\}$ and edges straight line segments and satisfying the following conditions:

a) None of the edges is horizontal.

b) If $\epsilon_{i}=+$ then $y_{i} \geqslant z$ for any $(i, z) \in T$. So, the tree goes under $\left(i, y_{i}\right)$.

c) If $\epsilon_{i}=-$ then $y_{i} \leqslant z$ for any $(i, z) \in T$. So, the tree goes over $\left(i, y_{i}\right)$.

d) If $\epsilon_{i}=+$ then there is at most one edge descending from $\left(i, y_{i}\right)$ and at most two edges ascending from $\left(i, y_{i}\right)$ and not on the same side.

$e)$ If $\epsilon_{i}=-$ then there is at most one edge ascending from $\left(i, y_{i}\right)$ and at most two edges descending from $\left(i, y_{i}\right)$ and not on the same side.

Two MCTs $T, T^{\prime}$ are isomorphic as MCTs if there is a graph isomorphism $T \cong T^{\prime}$ which sends $\left(i, y_{i}\right)$ to $\left(i, y_{i}^{\prime}\right)$ and so that corresponding edges have the same sign of their slopes.

Given a MCT $T$, there is a partial ordering on $[0, n]$ given by $i<_{T} j$ if the unique path from $\left(i, y_{i}\right)$ to $\left(j, y_{j}\right)$ in $T$ is monotonically increasing. Isomorphic MCTs give the same partial ordering by definition. Conversely, the partial ordering $<_{T}$ determines $T$ uniquely up to isomorphism since $T$ is the Hasse diagram of the partial ordering $<_{T}$. We sometimes decorate MCTs with leaves at vertices so that the result is trivalent, i.e., with three edges incident to each vertex. See, e.g., Figure 6 . The ends of these leaves are not considered to be vertices. In that case, each vertex with $\epsilon=+$ forms a "Y" and this pattern is vertically inverted for $\epsilon=-$. The position of the leaves is uniquely determined.

In Figure 6, the four vertices have coordinates $\left(0, y_{0}\right),\left(1, y_{1}\right),\left(2, y_{2}\right),\left(3, y_{3}\right)$ where the $y_{i}$ can be any real numbers such that $y_{0}<y_{1}<y_{2}<y_{3}$. This inequality defines an open subset of $\mathbb{R}^{4}$ which is called the region of this tree $T$. More generally, for any MCT $T$, the region of $T$, denoted $\mathcal{R}_{\epsilon}(T)$, is the set of all points $y \in \mathbb{R}^{n+1}$ with the property that there exists a mixed cobinary tree $T^{\prime}$ which is isomorphic to $T$ so that the vertex set of $T^{\prime}$ is $\left\{\left(i, y_{i}\right): i \in[0, n]\right\}$.

Theorem 35 ([IO13]). Let $n \in \mathbb{Z}_{\geqslant 0}$ and $\epsilon:[0, n] \rightarrow\{+,-\}$ be fixed. Then, for every $M C T$ $T$, the region $\mathcal{R}_{\epsilon}(T)$ is convex and nonempty. Furthermore, every point $y=\left(y_{0}, \ldots, y_{n}\right)$ in $\mathbb{R}^{n+1}$ with distinct coordinates lies in $\mathcal{R}_{\epsilon}(T)$ for a unique $T$ (up to isomorphism). In particular these regions are disjoint and their union is dense in $\mathbb{R}^{n+1}$. 


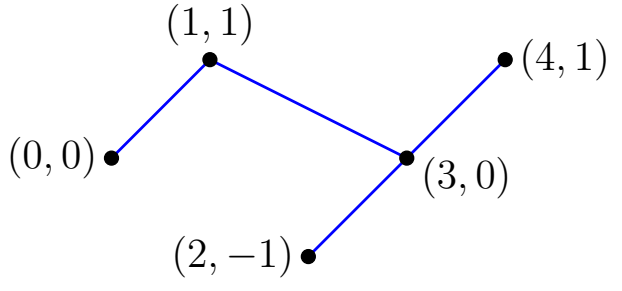

Figure 5: A MCT with $\epsilon_{1}=\epsilon_{2}=-$, $\epsilon_{3}=+$ and any value for $\epsilon_{0}, \epsilon_{4}$.

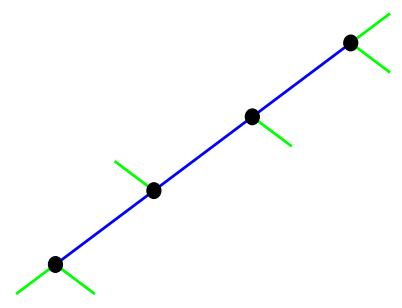

Figure 6: This MCT (in blue) has added green leaves showing that $\epsilon=$ $(-,+,-,-)$.

For a fixed $n$ and $\epsilon:[0, n] \rightarrow\{+,-\}$ we will construct a bijection between the set $\mathcal{T}_{\epsilon}$ of isomorphism classes of mixed cobinary trees with sign function $\epsilon$ and the set $\overrightarrow{\mathcal{D}}_{n, \epsilon}$ defined in Definition 19.

Let $\vec{d}=\left\{\vec{c}\left(i_{\ell}, j_{\ell}\right)\right\}_{\ell \in[n]} \in \overrightarrow{\mathcal{D}}_{n, \epsilon}$, and let $\vec{\gamma}_{\ell}$ be an element of the set $\vec{c}\left(i_{\ell}, j_{\ell}\right)$ of oriented curves with $\ell \in[n]$ so that each $\overrightarrow{\gamma_{\ell}}$ is a monotone curve from $i_{\ell}$ to $j_{\ell}$ and so that the oriented curves $\left\{\vec{\gamma}_{\ell}\right\}_{\ell \in[n]}$ are pairwise nonintersecting in their interiors. Let $p=(x, y)$ and $q=(x, z)$ be two distinct points on the union of these oriented curves where $q$ lies above $p$. The oriented diagram $\vec{d}$ defines a unique sequence of oriented curves $\overrightarrow{\lambda_{i_{1}}}, \ldots, \overrightarrow{\lambda_{i_{k}}}$ joining $p$ and $q$ where $\overrightarrow{\lambda_{i_{1}}} \subset \overrightarrow{\gamma_{i_{1}}}$ or $\overrightarrow{\lambda_{i_{k}}} \subset \overrightarrow{\gamma_{i_{k}}}$ as subsets of $\mathbb{R}^{2}$ and $\overrightarrow{\lambda_{i_{t}}}=\overrightarrow{\gamma_{i_{t}}}$ for all $t \in\{2, \ldots, k-1\}$ for some collection of oriented curves $\overrightarrow{\gamma_{i_{1}}}, \ldots, \overrightarrow{\gamma_{i_{k}}} \in\left\{\overrightarrow{\gamma_{\ell}}\right\}_{\ell \in[n]}$. We study the sequence $\overrightarrow{\lambda_{i_{1}}}, \ldots, \overrightarrow{\lambda_{i_{k}}}$ in the next lemma.

Lemma 36. In the notation of the previous paragraph, if $p=(x, y)$ and $q=(x, z)$ are two points on the union of the curves $\left\{\vec{\gamma}_{\ell}\right\}_{\ell \in[n]}$ where $q$ lies above $p$, then the sequence $\overrightarrow{\lambda_{i_{1}}}, \ldots, \overrightarrow{\lambda_{i_{k}}}$ forms an oriented path that is oriented from $p$ to $q$.

Proof. The proof will be by induction on the number $m$ of internal vertices appearing in the sequence $\overrightarrow{\lambda_{i_{1}}}, \ldots, \overrightarrow{\lambda_{i_{k}}}$. If $m=1$ with internal vertex $\left(x_{j}, 0\right)$ then $p$ and $q$ are connected by $\overrightarrow{\lambda_{i_{1}}}$ and $\overrightarrow{\lambda_{i_{2}}}$ where the former connects $p$ to $\left(x_{j}, 0\right)$ and the latter connects $\left(x_{j}, 0\right)$ back to $q$. Since the oriented curves $\left\{\vec{\gamma}_{\ell}\right\}_{\ell \in[n]}$ are pairwise nonintersecting, the oriented curve $\overrightarrow{\lambda_{i_{1}}}$ lies below $\overrightarrow{\lambda_{i_{2}}}$. Since $\vec{d} \in \overrightarrow{\mathcal{D}}_{n, \epsilon}$, we conclude that $\overrightarrow{\lambda_{i_{1}}}$ (resp., $\overrightarrow{\lambda_{i_{2}}}$ ) is oriented from $p$ to $\left(x_{j}, 0\right)$ (resp., from $\left(x_{j}, 0\right)$ to $q$ ).

Now suppose that $m \geqslant 2$ and the lemma holds for smaller $m$. There are two cases. Case 1: the sequence $\overrightarrow{\lambda_{i_{1}}}, \ldots, \overrightarrow{\lambda_{i_{k}}}$ lies entirely on one side of $p$ and $q$ (as in the case $m=1$ ). Case 2: the sequence $\overrightarrow{\lambda_{i_{1}}}, \ldots, \overrightarrow{\lambda_{i_{k}}}$ has internal vertices on both sides of $p$ and $q$.

Case 1: Suppose by symmetry that the sequence $\overrightarrow{\lambda_{i_{1}}}, \ldots, \overrightarrow{\lambda_{i_{k}}}$ lies entirely to the left of $p$ and $q$. Let $\left(x_{j}, 0\right)$ denote the internal vertex of $\overrightarrow{\lambda_{i_{1}}}, \ldots, \overrightarrow{\lambda_{i_{k}}}$ with the largest value of $x_{j}$. Thus all other internal vertices of $\overrightarrow{\lambda_{i_{1}}}, \ldots, \overrightarrow{\lambda_{i_{k}}}$ are weakly to the left of $\left(x_{j}, 0\right)$ and $p$ and $q$ are to the right of $\left(x_{j}, 0\right)$. Let $\overrightarrow{\lambda_{i_{1}}}, \ldots, \overrightarrow{\lambda_{i_{t}}}$ (resp., $\overrightarrow{\lambda_{i_{t+1}}}, \ldots, \overrightarrow{\lambda_{i_{k}}}$ ) denote the sequence of 
oriented curves connecting $p$ and $\left(x_{j}, 0\right)$ (resp., $\left(x_{j}, 0\right)$ and $q$ ).

We claim that $\left(x_{j}, 0\right)$ cannot be a local maximum of $\gamma$ with respect to the $x$-coordinate. Assume by symmetry that $\epsilon_{j}=-$. Then the curves determined by $\overrightarrow{\lambda_{i_{1}}}, \ldots, \overrightarrow{\lambda_{i_{t}}}$ and $\overrightarrow{\lambda_{i_{t+1}}}, \ldots, \overrightarrow{\lambda_{i_{k}}}$, respectively, are required to pass over $\left(x_{j}, 0\right)$, say at points $r=\left(x_{j}, y_{j}\right)$ and $s=\left(x_{j}, z_{j}\right)$, respectively. Here $s$ lies above $r$. Since $r$ and $s$ both lie above $\left(x_{j}, 0\right)$, by induction on $m$ we know that each of the two sequences of oriented curves joining $\left(x_{j}, 0\right)$ to $r$ and $\left(x_{j}, 0\right)$ to $s$ form oriented paths oriented away from $\left(x_{j}, 0\right)$. This contradicts the definition of $\overrightarrow{\mathcal{D}}_{n, \epsilon}$ since two oriented curves leaving a vertex to the left cannot both be oriented away from that vertex.

Now, by the maximality of $x_{j}$, we have that either $\overrightarrow{\lambda_{i_{1}}}$ connects $\left(x_{j}, 0\right)$ and $p$ or $\overrightarrow{\lambda_{i_{k}}}$ connects $\left(x_{j}, 0\right)$ and $q$. Without loss of generality, we assume that $\overrightarrow{\lambda_{i_{1}}}$ connects $\left(x_{j}, 0\right)$ and $p$. By the maximality of $x_{j}$, there is a unique point $r=\left(x_{j}, y_{j}\right)$ on the oriented curve $\overrightarrow{\lambda_{i_{k}}}$ that lies directly above $\left(x_{j}, 0\right)$. In particular, this means $\epsilon_{j}=-$. By abuse of notation, let $\overrightarrow{\lambda_{i_{2}}}, \ldots, \overrightarrow{\lambda_{i_{k}}}$ denote the sequence of oriented curves connecting $\left(x_{j}, 0\right)$ to $r$. By induction, we obtain that the sequence of curves $\overrightarrow{\lambda_{i_{2}}}, \ldots, \overrightarrow{\lambda_{i_{k}}}$ forms an oriented path going from $\left(x_{j}, 0\right)$ to $r$. Now since $\vec{d} \in \overrightarrow{\mathcal{D}}_{n, \epsilon}$ and since $\epsilon_{j}=-$, we have that $\overrightarrow{\lambda_{i_{1}}}$ must be oriented toward $\left(x_{j}, 0\right)$. This completes Case 1.

Case 2: Suppose that the sequence $\overrightarrow{\lambda_{i_{1}}}, \ldots, \overrightarrow{\lambda_{i_{k}}}$ lies on both sides of the vertical line containing $p$ and $q$. This means the sequence $\overrightarrow{\lambda_{i_{1}}}, \ldots, \overrightarrow{\lambda_{i_{k}}}$ contains a third point $r=(x, w)$ from the vertical line containing $p$ and $q$. By abuse of notation, we let $\overrightarrow{\lambda_{i_{1}}}, \ldots, \overrightarrow{\lambda_{i_{t}}}$ and $\overrightarrow{\lambda_{i_{t+1}}}, \ldots, \overrightarrow{\lambda_{i_{k}}}$, respectively, denote the unique sequences of curves connecting $p$ to $r$ and $r$ to $q$, respectively. There are the following three possible subcases:

a) the point $r$ lies between $p$ and $q$,

$b$ ) the point $r$ lies below both $p$ and $q$, and

c) the point $r$ lies above both $p$ and $q$.

Suppose we are in subcase $a$ ). By induction, the sequence $\overrightarrow{\lambda_{i_{1}}}, \ldots, \overrightarrow{\lambda_{i_{t}}}$ (resp., $\overrightarrow{\lambda_{i_{t+1}}}, \ldots$, $\overrightarrow{\lambda_{i_{k}}}$ ) forms an oriented path that goes from $p$ to $r$ (resp., from $r$ to $q$ ). This completes the proof of subcase $a$ ).

We now prove subcase $b$ ), and we omit the proof of subcase $c$ ) as it is very similar. By induction, the sequence $\overrightarrow{\lambda_{i_{1}}}, \ldots, \overrightarrow{\lambda_{i_{t}}}$ (resp. $, \overrightarrow{\lambda_{i_{t+1}}}, \ldots, \overrightarrow{\lambda_{i_{k}}}$ ) forms an oriented path that goes from $r$ to $p$ (resp., $r$ to $q$ ). Since the oriented curves $\left\{\vec{\gamma}_{\ell}\right\}_{\ell \in[n]}$ are pairwise nonintersecting and since there are two oriented curves oriented away from $r$, we must have that $r=$ $\left(x_{i}, 0\right) \in \mathcal{S}_{n, \epsilon}$ for some $i$. Moreover, since $p$ and $q$ lie above $r$, we know that $\epsilon_{i}=-$ However, this contradicts that $\vec{d} \in \overrightarrow{\mathcal{D}}_{n, \epsilon}$. This completes that proof.

Theorem 37. For each $\vec{d}=\left\{\vec{c}\left(i_{\ell}, j_{\ell}\right)\right\}_{\ell \in[n]} \in \overrightarrow{\mathcal{D}}_{n, \epsilon}$, let $\mathcal{R}(\vec{d})$ denote the set of all $y \in \mathbb{R}^{n+1}$ so that $y_{i}<y_{j}$ for any $\vec{c}(i, j)$ in $\vec{d}$. Then $\mathcal{R}(\vec{d})=\mathcal{R}_{\epsilon}(T)$ for a uniquely 
determined mixed cobinary tree $T \in \mathcal{T}_{\epsilon}$. Furthermore, this gives a bijection

$$
\overrightarrow{\mathcal{D}}_{n, \epsilon} \cong \mathcal{T}_{\epsilon}
$$

Proof. We first verify the existence of a mixed cobinary tree $T$ for every choice of $y \in$ $\mathcal{R}(\vec{d})$. Since the strand diagram is a tree, the vector $y$ is uniquely determined by $y_{0} \in \mathbb{R}$ and $y_{j_{\ell}}-y_{i_{\ell}}>0, \ell \in[n]$, which are arbitrary. Given such a $y$, we claim that the $n$ line segments $L_{\ell}$ in $\mathbb{R}^{2}$ connecting the pairs of points $\left(i_{\ell}, y_{i_{\ell}}\right),\left(j_{\ell}, y_{j_{\ell}}\right)$ meet only at their endpoints. If not then two of these line segments, say $L_{s}, L_{t}$, meet at some point $(a, b) \in \mathbb{R}^{2}$. This leads to a contradiction of Lemma 36 as follows. Let $\left\{\vec{\gamma}_{\ell}\right\}_{\ell \in[n]}$ with $\overrightarrow{\gamma_{\ell}} \in \vec{c}\left(i_{\ell}, j_{\ell}\right)$ for all $\ell \in[n]$ be monotone curves that are pairwise nonintersecting in their interiors. Let $p \in \overrightarrow{\gamma_{s}}$ and $q \in \overrightarrow{\gamma_{t}}$ be the points on those curves with $x$-coordinate $a$. By symmetry assume $p$ is below $q$. Let $\overrightarrow{\lambda_{w_{1}}}, \ldots, \overrightarrow{\lambda_{w_{k}}}$ denote the sequence of oriented curves connecting $p$ and $q$ so that $w_{1}=s$ and $w_{k}=t$. By Lemma 36, these oriented curves form an oriented path going from $p$ to $q$. By definition of the vector $y \in \mathcal{R}(\vec{d})$ we have $y_{i_{\ell}}<y_{j_{\ell}}$ for each $\ell=w_{1}, \ldots, w_{k}$. Then $b<y_{j_{s}}<y_{i_{t}}<b$ is a contradiction. So, $T$ is a linearly embedded tree. The lemma also implies that the tree $T$ lies above all negative vertices and below all positive vertices. The other parts of Definition 34 follow from the definition of an oriented strand diagram. Therefore $T \in \mathcal{T}_{\epsilon}$. Since this argument works for every $y \in \mathcal{R}(\vec{d})$, we see that $\mathcal{R}(\vec{d})=\mathcal{R}_{\epsilon}(T)$ as claimed.

A description of the inverse mapping $\mathcal{T}_{\epsilon} \rightarrow \overrightarrow{\mathcal{D}}_{n, \epsilon}$ is given as follows. Take any $\epsilon$ MCT $T$ and deform the tree by moving all vertices vertically to the subset $[0, n] \times 0$ on the $x$-axis and deforming the edges into curved arcs in such a way that they are always embedded in the plane with no vertical tangents and so that their interiors do not meet. The result is a collection of oriented curves representing an oriented strand diagram $\vec{d}$ with $\mathcal{R}(\vec{d})=\mathcal{R}_{\epsilon}(T)$. An explicit formula for these curves can be given using a formula similar to Equation 1.

It is clear that these are inverse mappings giving the desired bijection $\overrightarrow{\mathcal{D}}_{n, \epsilon} \cong \mathcal{T}_{\epsilon}$.

Example 38. The MCTs in Figures 5 and 6 above give the oriented strand diagrams:
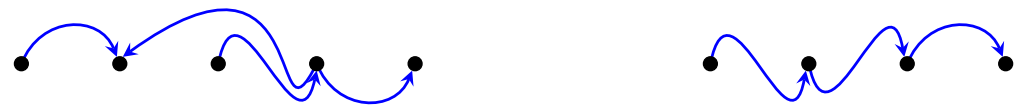

and the oriented strand diagram in Example 21 gives the MCT:
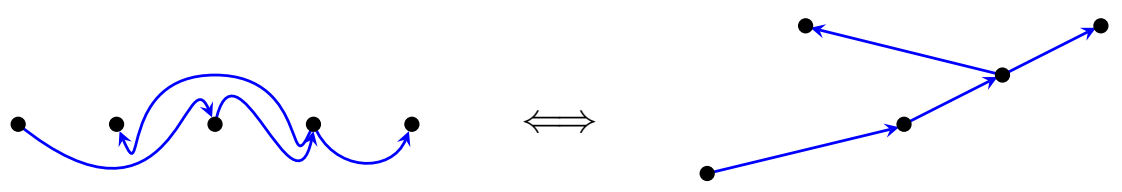
We now arrive at the proof of Theorem 22. This theorem follows from the fact that oriented diagrams belonging to $\overrightarrow{\mathcal{D}}_{n, \epsilon}$ can be regarded as mixed cobinary trees by Theorem 37.

Proof of Theorem 22. Let $f$ be the map c-mat $\left(Q_{\epsilon}\right) \rightarrow \overrightarrow{\mathcal{D}}_{n, \epsilon}$ induced by the map defined in Lemma 20, and let $g$ be the bijective map $\mathcal{T}_{\epsilon} \rightarrow \overrightarrow{\mathcal{D}}_{n, \epsilon}$ defined in Theorem 37. We will assert the existence of a map $h: \mathbf{c}$-mat $\left(Q_{\epsilon}\right) \rightarrow \mathcal{T}_{\epsilon}$ which fits into the diagram

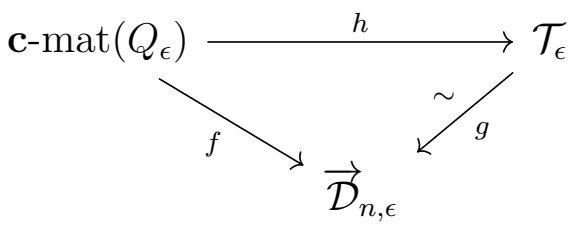

The theorem will follow after verifying that $h$ is a bijection and that $f=g \circ h$.

We will define two new notions of c-matrix, one for MCTs and one for oriented strand diagrams. Let $T \in \mathcal{T}_{\epsilon}$ with internal edges $\ell_{i}$ having endpoints $\left(i_{1}, y_{i_{1}}\right)$ and $\left(i_{2}, y_{i_{2}}\right)$. For each $\ell_{i}$, define the 'c-vector' of $\ell_{i}$ to be $c_{i}(T):=\sum_{i_{1}<j \leqslant i_{2}} \operatorname{sgn}\left(\ell_{i}\right) e_{j}$, where $\operatorname{sgn}\left(\ell_{i}\right)$ is the sign of the slope of $\ell_{i}$. Define $c(T)$ to be the 'c-matrix' of $T$ whose rows are the c-vectors $c_{i}(T)$. Now, let $\vec{d}=\left\{\vec{c}\left(i_{\ell}, j_{\ell}\right)\right\}_{\ell \in[n]} \in \overrightarrow{\mathcal{D}}_{n, \epsilon}$. For each oriented strand $\vec{c}\left(i_{\ell}, j_{\ell}\right)$, define the 'c-vector' of $\vec{c}\left(i_{\ell}, j_{\ell}\right)$ to be

$$
c_{\ell}(\vec{d}):=\left\{\begin{array}{lll}
\sum_{i_{\ell}<k \leqslant j_{\ell}} \operatorname{sgn}\left(\vec{c}\left(i_{\ell}, j_{\ell}\right)\right) e_{k} & : i_{\ell}<j_{\ell} \\
\sum_{j_{\ell}<k \leqslant i_{\ell}} \operatorname{sgn}\left(\vec{c}\left(i_{\ell}, j_{\ell}\right)\right) e_{k} & : & i_{\ell}>j_{\ell}
\end{array}\right.
$$

where $\operatorname{sgn}\left(\vec{c}\left(i_{\ell}, j_{\ell}\right)\right)$ is positive if $i_{\ell}<j_{\ell}$ and negative if $i_{\ell}>j_{\ell}$. Define $c(\vec{d})$ to be the 'c-matrix' of $\vec{d}$ whose rows are the c-vectors $c_{\ell}(\vec{d})$.

It is known that the notion of $\mathbf{c}$-matrix for MCTs coincides with the original notion of c-matrix defined in Section 2.1, and that there is a bijection between $\mathbf{c}$-mat $\left(Q_{\epsilon}\right)$ and $\mathcal{T}_{\epsilon}$ which preserves c-matrices (see [IO13, Remarks 2 and 4] for details). Thus, we have a bijective map $h: \mathbf{c}$-mat $\left(Q_{\epsilon}\right) \rightarrow \mathcal{T}_{\epsilon}$. On the other hand, the bijection $g: \mathcal{T}_{\epsilon} \rightarrow \overrightarrow{\mathcal{D}}_{n, \epsilon}$ defined in Theorem 37 also preserves c-matrices. The map $f: \mathbf{c}$-mat $\left(Q_{\epsilon}\right) \rightarrow \mathcal{T}_{\epsilon}$ This $\mathcal{T}_{\epsilon}$ should be $\overrightarrow{\mathcal{D}}_{n, \epsilon}$ preserves c-matrices by definition. Hence, we have $f=g \circ h$ and $f$ is a bijection, as desired.

Remark 39. For linearly-ordered quivers (those with $\epsilon=(+, \ldots,+)$ or $\epsilon=(-, \ldots,-)$ ), this bijection was established by the first and third authors in [GM15] using a different approach.

\section{Exceptional sequences and linear extensions}

In this section, we study CESs as linear extensions of certain posets. Our main result is a complete classification of these posets. 
Definition 40. Given a strand diagram $d$, we define $\mathcal{P}_{d}=\left(\left\{c\left(i_{\ell}, j_{\ell}\right)\right\}_{\ell \in[n]}, \leqslant\right)$ to be the poset whose elements are the strands of $d$ and where $c(k, \ell)$ covers $c(i, j)$, denoted by $c(i, j) \lessdot c(k, \ell)$, if and only if the strand $c(k, \ell)$ is clockwise from $c(i, j)$ and there does not exist another strand $c\left(i^{\prime}, j^{\prime}\right)$ distinct from $c(i, j)$ and $c(k, \ell)$ such that $c\left(i^{\prime}, j^{\prime}\right)$ is clockwise from $c(i, j)$ and counterclockwise from $c(k, \ell)$.

This construction defines a poset because any oriented cycle in the Hasse diagram of $\mathcal{P}_{d}$ arises from a cycle in the graph determined by $d$. Since the graph determined by $d$ is a tree, it has no cycles. In Figure 7 , we show a diagram $d \in \mathcal{D}_{4, \epsilon}$ where $\epsilon:=(+,+,-,+,-)$ and its poset $\mathcal{P}_{d}$.

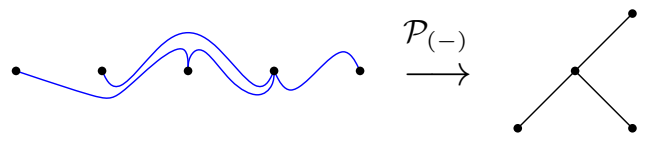

Figure 7: A diagram and its poset.
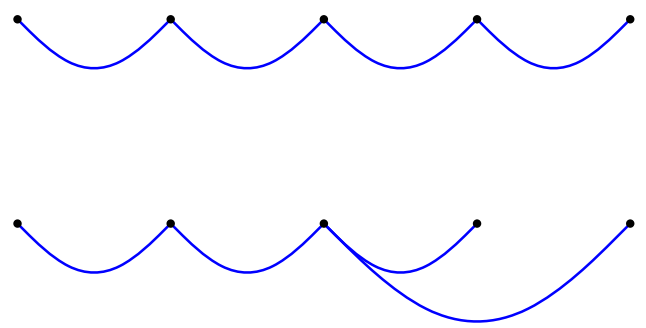

Figure 8: Two diagrams with the same poset.

In general, the map $\mathcal{D}_{n, \epsilon} \rightarrow \mathscr{P}\left(\mathcal{D}_{n, \epsilon}\right):=\left\{\mathcal{P}_{d}: d \in \mathcal{D}_{n, \epsilon}\right\}$ is not injective. For instance, each of the two diagrams in Figure 8 have $\mathcal{P}_{d}=\mathbf{4}$ where 4 denotes the linearly-ordered poset with 4 elements. It is thus natural to ask which posets are obtained from strand diagrams.

Our next result describes the posets arising from diagrams in $\mathcal{D}_{n, \epsilon}$ where $\epsilon=(-, \ldots,-)$ or $\epsilon=(+, \ldots,+)$. Before we state it, we remark that diagrams in $\mathcal{D}_{n, \epsilon}$ where $\epsilon=$ $(-, \ldots,-)$ or $\epsilon=(+, \ldots,+)$ can be regarded as chord diagrams. ${ }^{2}$ Figure 9 gives an example of this identification. Under this identification, the term strand is synonymous with chord.

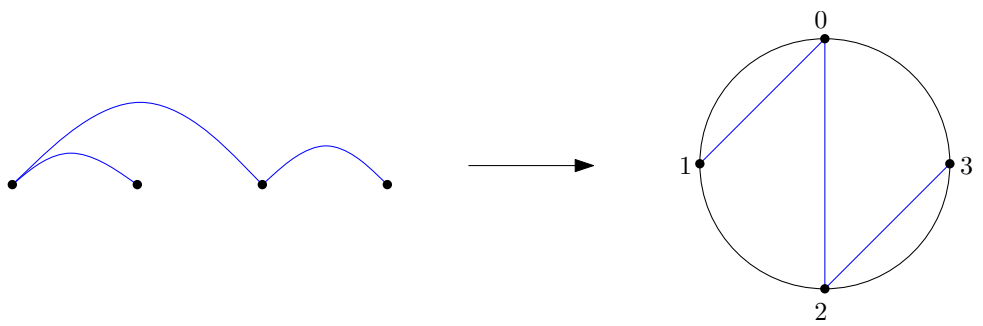

Figure 9: The identification between strand diagrams and chord diagrams.

Let $d \in \mathcal{D}_{n, \epsilon}$ where $\epsilon=(-, \ldots,-)$ or $\epsilon=(+, \ldots,+)$. Let $c(i, j)$ be a strand of d. There is an obvious action of $\mathbb{Z} /(n+1) \mathbb{Z}$ on chord diagrams. Let $\tau \in \mathbb{Z} /(n+1) \mathbb{Z}$

\footnotetext{
${ }^{2}$ These noncrossing trees embedded in a disk with vertices lying on the boundary have been studied by Araya in [Ara13], Goulden and Yong in [GY02], and the first and third authors in [GM15].
} 
denote a generator and define $\tau c(i, j):=c(i-1, j-1)$ and $\tau^{-1} c(i, j):=c(i+1, j+1)$ where we consider $i \pm 1$ and $j \pm 1 \bmod n+1$. We also define $\tau d:=\left\{\tau c\left(i_{\ell}, j_{\ell}\right)\right\}_{\ell \in[n]}$ and $\tau^{-1} d:=\left\{\tau^{-1} c\left(i_{\ell}, j_{\ell}\right)\right\}_{\ell \in[n]}$. The next lemma, which is easily verified, shows that the order-theoretic properties of CECs are invariant under the action of $\tau^{ \pm 1}$.

Lemma 41. Let $d \in \mathcal{D}_{n, \epsilon}$ where $\epsilon=(-, \ldots,-)$ or $\epsilon=(+, \ldots,+)$. Then we have the following isomorphisms of posets $\mathcal{P}_{d} \cong \mathcal{P}_{\tau d}$ and $\mathcal{P}_{d} \cong \mathcal{P}_{\tau^{-1} d}$.

Remark 42. One may interpret the action of $\tau$ as the Auslander-Reiten translation on the orbit category $D^{b}\left(\mathbb{k} Q_{\epsilon}\right) /[1]$ where $D^{b}\left(\mathbb{k} Q_{\epsilon}\right)$ is the bounded derived category of the module category of $\mathbb{k} Q_{\epsilon}$. A similar interpretation is given in [Ara13, Section 4].

Theorem 43. Let $\epsilon=(-, \ldots,-)$ or let $\epsilon=(+, \ldots,+)$. Then a poset $\mathcal{P} \in \mathscr{P}\left(\mathcal{D}_{n, \epsilon}\right)$ if and only if

i) each $x \in \mathcal{P}$ has at most two covers and covers at most two elements,

ii) the underlying graph of the Hasse diagram of $\mathcal{P}$ has no cycles,

iii) the Hasse diagram of $\mathcal{P}$ is connected.

We may equivalently define exceptional sequences and exceptional collections in terms of the derived category. We use this alternative formulation and Theorem 43 to show that the posets in $\mathscr{P}\left(\mathcal{D}_{n, \epsilon^{\prime}}\right)$ where $\epsilon^{\prime}$ is any element of $\{+,-\}^{n+1}$ have the same classification as those belonging to $\mathscr{P}\left(\mathcal{D}_{n, \epsilon}\right)$ where $\epsilon=(-, \ldots,-)$.

We say that an object $X \in D^{b}(\mathbb{k} Q)$ is exceptional if $\operatorname{Hom}_{D^{b}(\mathbb{k} Q)}(X, X) \cong \mathbb{k}$ and $\operatorname{Hom}_{D^{b}(\mathbb{k} Q)}(X[\ell], X)=0$ for any integer $\ell \neq 0$. A sequence $\left(X_{1}, X_{2}, \ldots, X_{r}\right)$ of exceptional objects of $D^{b}(\mathbb{k} Q)$ is called an exceptional sequence if for each $i<j$ one has $\operatorname{Hom}_{D^{b}(\mathbb{k} Q)}\left(X_{j}[\ell], X_{i}\right)=0$ for all integers $\ell$. An exceptional collection is a set $\left\{X_{1}, X_{2}, \ldots, X_{r}\right\}$ of exceptional objects that can be ordered in such a way that they define an exceptional sequence.

It is known that for any indecomposable object $E \in D^{b}\left(\mathbb{k} Q_{\epsilon}\right)$, there is a unique integer $\ell$ and a unique indecomposable $\mathbb{k} Q_{\epsilon}$-module $X$ such that $X \cong E[\ell]$. Moreover, if $\left(X_{1}, X_{2}, \ldots, X_{r}\right)$ is an exceptional sequence in $D^{b}\left(\mathbb{k} Q_{\epsilon}\right)$, then $\left(X_{1}\left[\ell_{1}\right], X_{2}\left[\ell_{2}\right], \ldots, X_{r}\left[\ell_{r}\right]\right)$ is an exceptional sequence in $D^{b}\left(\mathbb{k} Q_{\epsilon}\right)$ for any integers $\ell_{1}, \ell_{2}, \ldots, \ell_{r}$. It is also well-known that for any two $\epsilon, \epsilon^{\prime} \in\{+,-\}^{n+1}$, the derived categories $D^{b}\left(\mathbb{k} Q_{\epsilon}\right)$ and $D^{b}\left(\mathbb{k} Q_{\epsilon^{\prime}}\right)$ are triangle-equivalent (see [Hap88]). Thus any exceptional sequence of $Q_{\epsilon^{\prime}}$ where $\epsilon^{\prime}$ is any element of $\{+,-\}^{n+1}$ may be identified with an exceptional sequence of $Q_{\epsilon}$ where $\epsilon=$ $(-, \ldots,-) \in\{+,-\}^{n+1}$. These facts were already observed in [Ara13, Remark 2.2]. We obtain the following corollary of Theorem 43.

Corollary 44. Given any $\epsilon \in\{+,-\}^{n+1}$, a poset $\mathcal{P} \in \mathscr{P}\left(\mathcal{D}_{n, \epsilon}\right)$ if and only if $\mathcal{P}$ satisfies the conditions appearing in Theorem 43.

Proof of Theorem 43. Let $\mathcal{P}_{d} \in \mathscr{P}\left(\mathcal{D}_{n, \epsilon}\right)$. By definition, $\mathcal{P}_{d}$ satisfies $i$ ) and $\left.i i\right)$. It is also clear that the Hasse diagram of $\mathcal{P}_{d}$ is connected since the graph determined by $d$ is connected.

To prove the converse, we proceed by induction on the number of elements of $\mathcal{P}$ where $\mathcal{P}$ is a poset satisfying conditions $i$ ), $i i), i i i)$. If $\# \mathcal{P}=1$, then $\mathcal{P}$ is the unique poset with 
one element and $\mathcal{P}=\mathcal{P}_{d}$ where $d$ is the unique chord diagram with a single chord in a disk with exactly two boundary vertices. Assume that for any poset $\mathcal{P}$ satisfying conditions $i), i i), i i i)$ with $\# \mathcal{P}=r$ for any positive integer $r<n+1$ there exists a chord diagram $d$ such that $\mathcal{P}=\mathcal{P}_{d}$. Let $\mathcal{Q}$ be a poset satisfying the above conditions where $\# \mathcal{Q}=n+1$, and let $x \in \mathcal{Q}$ be a maximal element. We know $x$ covers either one or two elements of $\mathcal{Q}$.

Assume $x$ covers two elements $y, z \in \mathcal{Q}$. Since the Hasse diagram of $\mathcal{Q}$ has no cycles, we have that $\mathcal{Q}-\{x\}=\mathcal{Q}_{1}+\mathcal{Q}_{2}$ where $y \in \mathcal{Q}_{1}, z \in \mathcal{Q}_{2}$, and $\mathcal{Q}_{i}$ satisfies $i$ ), ii), iii) for $i \in[2]$. By induction, there exist positive integers $k_{1}, k_{2}$ satisfying $k_{1}+k_{2}=n$ and chord diagrams $d_{i}=\left\{c_{i}\left(i_{\ell}, j_{\ell}\right)\right\}_{\ell \in\left[k_{i}\right]} \in \mathcal{D}_{k_{i}, \epsilon^{(i)}}$ where $\mathcal{Q}_{i}=\mathcal{P}_{d_{i}}$ for $i \in[2]$ and where $\epsilon^{(i)} \in\{+,-\}^{k_{i}+1}$ has all of its entries equal to the corresponding entries of $\epsilon$. In addition, we know that $y$ (resp., $z$ ) is covered by at most one element of $\mathcal{Q}_{1}$ (resp., $\mathcal{Q}_{2}$ ). This means that the chord corresponding to $y$ (resp., $z$ ) in $d_{1}$ (resp., $d_{2}$ ) has an endpoint such that the chord is not counterclockwise from any other chord in $d_{1}$ (resp., $d_{2}$ ) about that endpoint.

By Lemma 41, we can assume that the chord corresponding to $y \in \mathcal{Q}_{1}$ (resp., $z \in \mathcal{Q}_{2}$ ) is $c_{1}\left(i(y), k_{1}\right) \in d_{1}$ for some $i(y) \in\left[0, k_{1}-1\right]$ and that $c_{1}\left(i(y), k_{1}\right)$ is not counterclockwise from any other chord of $d_{1}$ about $k_{1}$ (resp., $c_{2}\left(j(z), k_{2}\right) \in d_{2}$ for some $j(z) \in\left[0, k_{2}-1\right]$ and that $c_{2}\left(j(y), k_{2}\right)$ is not counterclockwise from any other chord of $d_{2}$ about $\left.k_{2}\right)$. Define $d_{1} \sqcup d_{2}:=\left\{c^{\prime}\left(i_{\ell}^{\prime}, j_{\ell}^{\prime}\right)\right\}_{\ell \in[n]}$ to be the chord diagram in the disk with $n+2$ boundary vertices as follows (see Figure 10):

$$
c^{\prime}\left(i_{\ell}^{\prime}, j_{\ell}^{\prime}\right):= \begin{cases}c_{1}\left(i_{\ell}, j_{\ell}\right) & : \text { if } \ell \in\left[k_{1}\right] \\ \tau^{-\left(k_{1}+1\right)} c_{2}\left(i_{\ell-k_{1}}, j_{\ell-k_{1}}\right) & : \text { if } \ell \in\left[k_{1}+1, n\right] .\end{cases}
$$
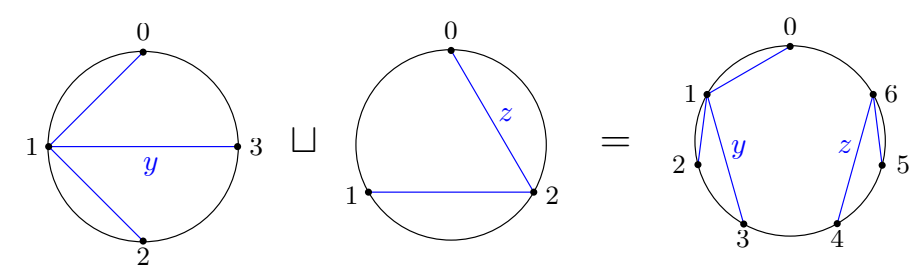

Figure 10: An example of $d_{1} \sqcup d_{2}$ with $k_{1}=3$ and $k_{2}=2$ so that $n=k_{1}+k_{2}=5$.

Define $c^{\prime}\left(i_{n+1}^{\prime}, j_{n+1}^{\prime}\right):=c\left(k_{1}, n+1\right)$ and then $d:=\left\{c^{\prime}\left(i_{\ell}^{\prime}, j_{\ell}^{\prime}\right)\right\}_{\ell \in[n+1]}$ satisfies $\left.\left.\left.i\right), i i\right), i i i\right)$, and $\mathcal{Q}=\mathcal{P}_{d}$.

Next, assume $x$ covers only the element $y \in \mathcal{Q}$. In this case, the Hasse diagram of $\mathcal{Q}-\{x\}$ is connected. By induction, the poset $\mathcal{Q}-\{x\}=\mathcal{P}_{d}$ for some diagram $d=\left\{c\left(i_{\ell}, j_{\ell}\right)\right\}_{\ell \in[n]} \in \mathcal{D}_{n, \epsilon}$ where we assume $i_{\ell}<j_{\ell}$. Let $y=c(i(y), j(y)) \in \mathcal{Q}-\{x\}$ with $i(y)<j(y)$ denote the unique element that is covered by $x$ in $\mathcal{Q}$. Note that $y$ is covered by at most one element of $\mathcal{Q}-\{x\}$. This means that by applying $\tau$ sufficiently many times to $d$ and using Lemma 41, we can assume that there are no chords in $d$ that are clockwise from $c(i(y), j(y))$ about $i(y)$.

We regard $d$ as an element of $\mathcal{D}_{n+1, \epsilon^{\prime}}$ by replacing it with $\widetilde{d}:=\left\{c^{\prime}\left(i_{\ell}^{\prime}, j_{\ell}^{\prime}\right)\right\}_{\ell \in[n]} \in \mathcal{D}_{n+1, \epsilon^{\prime}}$ as follows (see Figure 11): 


$$
c^{\prime}\left(i_{\ell}^{\prime}, j_{\ell}^{\prime}\right):= \begin{cases}c\left(i_{\ell}, j_{\ell}+1\right) & : \text { if } i_{\ell} \leqslant i(y) \text { and } j(y) \leqslant j_{\ell}, \\ \tau^{-1} c\left(i_{\ell}, j_{\ell}\right) & : \text { if } i(y) \leqslant i_{\ell}, \\ c\left(i_{\ell}, j_{\ell}\right) & : \text { otherwise. }\end{cases}
$$

Figure 11: An example of $\widetilde{d}$ with $n=6$.

Here $j_{\ell}+1$ is calculated $\bmod n+2$. Additionally, we are using that $i_{\ell}<j_{\ell}$ in the definition of $\widetilde{d}$.

Now define $c^{\prime}\left(i_{n+1}^{\prime}, j_{n+1}^{\prime}\right):=c(i(y), i(y)+1)$ and put $d^{\prime}:=\left\{c^{\prime}\left(i_{\ell}^{\prime}, j_{\ell}^{\prime}\right)\right\}_{\ell \in[n+1]}$. As $\mathcal{Q}-\{x\}$ satisfies $i), i i)$, and $i i i)$, it is clear that the resulting chord diagram $d^{\prime}$ also satisfies $\left.\left.i\right), i i\right)$, and $i i i$ ), and that $\mathcal{P}=\mathcal{P}_{d^{\prime}}$.

Let $\mathcal{P}$ be a finite poset with $m=\# \mathcal{P}$. Let $f: \mathcal{P} \rightarrow \mathbf{m}$ be an injective, order-preserving map (i.e., $x \leqslant y$ implies $f(x) \leqslant f(y)$ for all $x, y \in \mathcal{P}$ ) where $\mathbf{m}$ is the linearly-ordered poset with $m$ elements. We call $f$ a linear extension of $\mathcal{P}$. We denote the set of linear extensions of $\mathcal{P}$ by $\mathscr{L}(\mathcal{P})$.

Theorem 45. Let $d=\left\{c\left(i_{\ell}, j_{\ell}\right)\right\}_{\ell \in[n]} \in \mathcal{D}_{n, \epsilon}$ and let $\bar{\xi}_{\epsilon}$ denote the corresponding complete exceptional collection. Let $C E S\left(\bar{\xi}_{\epsilon}\right)$ denote the set of CESs that can be formed using only the representations appearing in $\bar{\xi}_{\epsilon}$. Then the map $\chi: C E S\left(\bar{\xi}_{\epsilon}\right) \rightarrow \mathscr{L}\left(\mathcal{P}_{d}\right)$ defined by $\left(X_{i_{1}, j_{1}}^{\epsilon}, \ldots, X_{i_{n}, j_{n}}^{\epsilon}\right) \stackrel{\chi_{2}}{\longmapsto}\left\{\left(c\left(i_{\ell}, j_{\ell}\right), n+1-\ell\right)\right\}_{\ell \in[n]} \stackrel{\chi_{1}}{\longmapsto}\left(f\left(c\left(i_{\ell}, j_{\ell}\right)\right):=n+1-\ell\right)$ is a bijection.

Proof. Let $\chi_{2}: \operatorname{CES}\left(\bar{\xi}_{\epsilon}\right) \rightarrow \hat{\mathcal{D}}_{n, \epsilon}$ be the restriction of the domain of the map $\widetilde{\Phi}_{\epsilon}$ to $\operatorname{CES}\left(\bar{\xi}_{\epsilon}\right)$. It follows that $\chi_{2}: \operatorname{CES}\left(\bar{\xi}_{\epsilon}\right) \rightarrow \chi_{2}\left(\operatorname{CES}\left(\bar{\xi}_{\epsilon}\right)\right)$ is a bijection by Theorem 16. Thus it is enough to prove that $\chi_{1}: \chi_{2}\left(\operatorname{CES}\left(\bar{\xi}_{\epsilon}\right)\right) \rightarrow \mathscr{L}\left(\mathcal{P}_{d}\right)$ is a bijection.

First, we show that $\chi_{1}(\hat{d}) \in \mathscr{L}\left(\mathcal{P}_{d}\right)$ for any $\hat{d} \in \chi_{2}\left(\operatorname{CES}\left(\bar{\xi}_{\epsilon}\right)\right)$. Let $\hat{d} \in \chi_{2}\left(\operatorname{CES}\left(\bar{\xi}_{\epsilon}\right)\right)$ and let $f:=\chi_{1}(\hat{d})$. Since the strand-labeling of $\hat{d}$ is good, if $\left(c_{1}, \ell_{1}\right)$ and $\left(c_{2}, \ell_{2}\right)$ are two labeled strands of $\hat{d}$ satisfying $c_{1} \leqslant c_{2}$, then $f\left(c_{1}\right)=\ell_{1} \leqslant \ell_{2}=f\left(c_{2}\right)$. Thus $f$ is order-preserving. As the strands of $\hat{d}$ are bijectively labeled by $[n]$, we have that $f$ is bijective so $f \in \mathscr{L}\left(\mathcal{P}_{d}\right)$.

Next, define a map

$$
\begin{aligned}
\mathscr{L}\left(\mathcal{P}_{d}\right) & \stackrel{\varphi}{\longrightarrow} \chi_{2}\left(\operatorname{CES}\left(\bar{\xi}_{\epsilon}\right)\right) \\
f & \longmapsto\left\{\left(c\left(i_{\ell}, j_{\ell}\right), f\left(c\left(i_{\ell}, j_{\ell}\right)\right)\right)\right\}_{\ell \in[n]}
\end{aligned}
$$

To see that $\varphi(f) \in \chi_{2}\left(\operatorname{CES}\left(\bar{\xi}_{\epsilon}\right)\right)$ for any $f \in \mathscr{L}\left(\mathcal{P}_{d}\right)$, consider two labeled strands $\left(c_{1}, f\left(c_{1}\right)\right)$ and $\left(c_{2}, f\left(c_{2}\right)\right)$ belonging to $\varphi(f)$ where $c_{1} \leqslant c_{2}$. Since $f$ is order-preserving, 
$f\left(c_{1}\right) \leqslant f\left(c_{2}\right)$. Thus the strand-labeling of $\varphi(f)$ is good so $\varphi(f) \in \hat{\mathcal{D}}_{n, \epsilon}$. Moreover, it is clear that the labeled diagram $\varphi(f)$ corresponds to a complete exceptional sequence formed using exactly the representations from $\bar{\xi}_{\epsilon}$. Thus $\varphi(f) \in \chi_{2}\left(\operatorname{CES}\left(\bar{\xi}_{\epsilon}\right)\right)$.

Lastly, we have that

$$
\chi_{1}(\varphi(f))=\chi_{1}\left(\left\{\left(c\left(i_{\ell}, j_{\ell}\right), f\left(c\left(i_{\ell}, j_{\ell}\right)\right)\right)\right\}_{\ell \in[n]}\right)=f
$$

and

$$
\varphi\left(\chi_{1}\left(\left\{\left(c\left(i_{\ell}, j_{\ell}\right), \ell\right)\right\}_{\ell \in[n]}\right)\right)=\varphi\left(f\left(c\left(i_{\ell}, j_{\ell}\right)\right):=\ell\right)=\left\{\left(c\left(i_{\ell}, j_{\ell}\right), \ell\right)\right\}_{\ell \in[n]}
$$

so $\varphi=\chi_{1}^{-1}$. Thus $\chi_{1}$ is a bijection.

\section{Applications}

Here we showcase some interesting results that follow easily from our main theorems.

\subsection{Reddening sequences}

In [Kel12], Keller proves that for any quiver $Q$, any two reddening mutation sequences applied to $\widehat{Q}$ produce isomorphic ice quivers. As mentioned in [Kel13], his proof is highly dependent on representation theory and geometry, but the statement is purely combinatorial — we give a combinatorial proof of this result for type $\mathbb{A}_{n}$ quivers $Q_{\epsilon}$.

Let $R \in E G(\widehat{Q})$. A mutable vertex $i \in R_{0}$ is called green if there are no arrows $j \rightarrow i$ in $R$ with $j \in[n+1, m]$. Otherwise, $i$ is called red. A sequence of mutations $\mu_{i_{r}} \circ \cdots \circ \mu_{i_{1}}$ is reddening if all mutable vertices of the quiver $\mu_{i_{r}} \circ \cdots \circ \mu_{i_{1}}(\widehat{Q})$ are $\operatorname{red}^{3}$. Recall that an isomorphism of quivers that fixes the frozen vertices is called a frozen isomorphism. We now state the theorem.

Theorem 46. If $\mu_{i_{r}} \circ \cdots \circ \mu_{i_{1}}$ and $\mu_{j_{s}} \circ \cdots \circ \mu_{j_{1}}$ are two reddening sequences of $\widehat{Q}_{\epsilon}$ for some $\epsilon \in\{+,-\}^{n+1}$, then there is a frozen isomorphism $\mu_{i_{r}} \circ \cdots \circ \mu_{i_{1}}\left(\widehat{Q}_{\epsilon}\right) \cong \mu_{j_{s}} \circ \cdots \circ \mu_{j_{1}}\left(\widehat{Q}_{\epsilon}\right)$.

Proof. Let $\mu_{i_{r}} \circ \cdots \circ \mu_{i_{1}}$ be any reddening sequence. Denote by $\bar{C}$ the c-matrix of $\mu_{i_{r}} \circ$ .. $\circ \mu_{i_{1}}\left(\widehat{Q}_{\epsilon}\right)$. By Theorem $22, \bar{C}$ corresponds to an oriented strand diagram $\overrightarrow{d_{\bar{C}}} \in \overrightarrow{\mathcal{D}}_{n, \epsilon}$ with all strands of the form $\vec{c}(j, i)$ for some $i$ and $j$ satisfying $i<j$. Since all strands of $\overrightarrow{d_{\bar{C}}}$ are oriented to the left and since $\overrightarrow{d_{\bar{C}}}$ satisfies Definition 19 , we conclude that $\overrightarrow{d_{\bar{C}}}=$ $\{\vec{c}(i, i-1)\}_{i \in[n]}$ and $\bar{C}=-I_{n}$. Since c-matrices are in bijection with ice quivers in $E G\left(\widehat{Q}_{\epsilon}\right)$ and since $\breve{Q}_{\epsilon}$ is an ice quiver in $E G\left(\widehat{Q}_{\epsilon}\right)$ whose c-matrix is $-I_{n}$, we obtain the desired result.

\subsection{Noncrossing partitions and exceptional sequences}

In this section, we give a combinatorial proof of Ingalls' and Thomas' result that complete exceptional sequences are in bijection with maximal chains in the lattice of noncrossing

\footnotetext{
${ }^{3}$ By abuse of notation, we write $\mu_{i_{r}} \circ \cdots \circ \mu_{i_{1}}(\widehat{Q})$ to denote $\left(\mu_{i_{r}} \circ \cdots \circ \mu_{i_{1}}\left(Q^{\prime}\right), F\right)$
} 
partitions [IT09]. We remark that their result is more general than that which we present here. Throughout this section, we assume that $Q_{\epsilon}$ has $\epsilon=(-, \ldots,-)$ and we regard the strand diagrams of $Q_{\epsilon}$ as chord diagrams.

A partition of $[n]$ is a collection $\pi=\left\{B_{\alpha}\right\}_{\alpha \in I} \in 2^{[n]}$ of subsets of $[n]$ called blocks that are nonempty, pairwise disjoint, and whose union is $[n]$. We denote the lattice of set partitions of $[n]$, ordered by refinement, by $\Pi_{n}$. A set partition $\pi=\left\{B_{\alpha}\right\}_{\alpha \in I} \in \Pi_{n}$ is called noncrossing if for any $i<j<k<\ell$ where $i, k \in B_{\alpha_{1}}$ and $j, \ell \in B_{\alpha_{2}}$, one has $B_{\alpha_{1}}=B_{\alpha_{2}}$. We denote the lattice of noncrossing partitions of $[n]$ by $N C^{\mathbb{A}}(n)$.

Label the vertices of a convex $n$-gon $\mathcal{S}$ with elements of $[n]$ so that reading the vertices of $\mathcal{S}$ counterclockwise determines an increasing sequence mod $n$. We can thus regard $\pi=\left\{B_{\alpha}\right\}_{\alpha \in I} \in N C^{\mathbb{A}}(n)$ as a collection of convex hulls $B_{\alpha}$ of vertices of $\mathcal{S}$ where $B_{\alpha}$ has empty intersection with any other block $B_{\alpha^{\prime}}$.

Let $n=5$. The following partitions all belong to $\Pi_{5}$, but only $\pi_{1}, \pi_{2}, \pi_{3} \in N C^{\mathbb{A}}(5)$.

$$
\begin{array}{ll}
\pi_{1}=\{\{1\},\{2,4,5\},\{3\}\}, & \pi_{2}=\{\{1,4\},\{2,3\},\{5\}\}, \\
\pi_{3}=\{\{1,2,3\},\{4,5\}\}, & \pi_{4}=\{\{1,3,4\},\{2,5\}\}
\end{array}
$$

Below we represent the partitions $\pi_{1}, \ldots, \pi_{4}$ as convex hulls of sets of vertices of a convex pentagon. We see from this representation that $\pi_{4} \notin N C^{\mathbb{A}}(5)$.

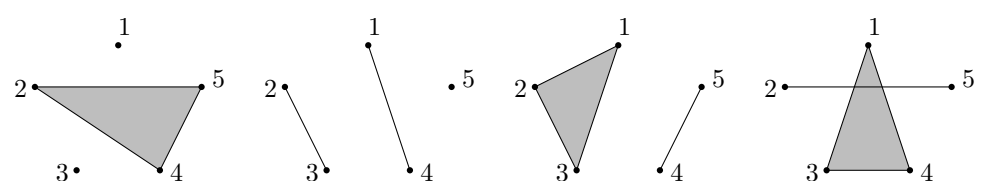

Theorem 47. Let $k \in[n]$. There is a bijection between $\hat{\mathcal{D}}_{k, \epsilon}$ and the following chains in $N C^{\mathbb{A}}(n+1)$

$$
\left\{\left(\pi_{1}=\{\{i\}\}_{i \in[n+1]}, \pi_{2}, \ldots, \pi_{k+1}\right): \begin{array}{c}
\pi_{j}=\left(\pi_{j-1} \backslash\left\{B_{\alpha}, B_{\beta}\right\}\right) \sqcup\left\{B_{\alpha} \sqcup B_{\beta}\right\} \\
\text { for some } B_{\alpha} \neq B_{\beta} \text { in } \pi_{j-1}
\end{array}\right\} .
$$

In particular, when $k=n$, there is a bijection between $\hat{\mathcal{D}}_{n, \epsilon}$ and maximal chains in $N C^{\mathbb{A}}(n+1)$. We remark that each chain described above is saturated (i.e., each inequality appearing in $\{\{i\}\}_{i \in[n+1]}<\pi_{1}<\cdots<\pi_{k}$ is a covering relation).

Proof. Let $\hat{d}=\left\{\left(c\left(i_{\ell}, j_{\ell}\right), \ell\right)\right\}_{\ell \in[k]} \in \hat{\mathcal{D}}_{k, \epsilon}$. Define $\pi_{\hat{d}, 1}:=\{\{i\}\}_{i \in[n+1]} \in \Pi_{n+1}$. Next, define $\pi_{\hat{d}, 2}:=\left(\pi_{\hat{d}, 1} \backslash\left\{\left\{i_{1}+1\right\},\left\{j_{1}+1\right\}\right\}\right) \sqcup\left\{\left\{i_{1}+1, j_{1}+1\right\}\right\}$. Now assume that $\pi_{\hat{d}, s}$ has been defined for some $s \in[k]$. Define $\pi_{\hat{d}, s+1}$ to be the partition obtained by merging the blocks of $\pi_{\hat{d}, s}$ containing $i_{s}+1$ and $j_{s}+1$. Now define $f(\hat{d}):=\left(\pi_{\hat{d}, 1}, \ldots, \pi_{\hat{d}, k+1}\right)$.

It is clear that $f(\hat{d})$ is a chain in $\Pi_{n+1}$ with the desired property as $\pi_{1} \lessdot \pi_{2}$ in $\Pi_{n+1}$ if and only if $\pi_{2}$ is obtained from $\pi_{1}$ by merging exactly two distinct blocks of $\pi_{1}$. To see that each $\pi_{\hat{d}, s} \in N C^{\mathbb{A}}(n+1)$, suppose a crossing of two blocks occurs in a partition appearing in $f(\hat{d})$. Let $\pi_{\hat{d}, s}$ be the smallest partition of $f(\hat{d})$ (with respect to the partial order on set partitions) with two blocks, $B_{1}$ and $B_{2}$, crossing. Without loss of generality, we assume that $B_{2} \in \pi_{\hat{d}, s}$ is obtained by merging the blocks $B_{\alpha_{1}}, B_{\alpha_{2}} \in \pi_{\hat{d}, s-1}$ containing $i_{s-1}+1$ and 
$j_{s-1}+1$, respectively. This means that $c\left(i_{s-1}, j_{s-1}\right) \in \hat{d}$ and $c\left(i_{s-1}, j_{s-1}\right)$ crosses at least one other chord of $\hat{d}$. This contradicts that $\hat{d} \in \hat{\mathcal{D}}_{k, \epsilon}$. Thus $f(\hat{d})$ is a chain in $N C^{\mathbb{A}}(n+1)$ with the desired property.

Next, we define a map $g$ that is the inverse of $f$. Let

$$
\mathcal{C}=\left(\pi_{1}=\{\{i\}\}_{i \in[n+1]}, \pi_{2}, \ldots, \pi_{k+1}\right) \in\left(N C^{\mathbb{A}}(n+1)\right)^{k+1}
$$

be a chain where each partition in $\mathcal{C}$ satisfies $\pi_{j}=\left(\pi_{j-1} \backslash\left\{B_{\alpha}, B_{\beta}\right\}\right) \sqcup\left\{B_{\alpha} \sqcup B_{\beta}\right\}$ for some $B_{\alpha} \neq B_{\beta}$ in $\pi_{j-1}$. As $\pi_{2}=\left(\pi_{1} \backslash\left\{\left\{s_{1}\right\},\left\{t_{1}\right\}\right\}\right) \sqcup\left\{\left\{s_{1}, t_{1}\right\}\right\}$ for some $s_{1}, t_{1} \in[n+1]$, define $c\left(i_{1}, j_{1}\right):=c\left(s_{1}-1, t_{1}-1\right)$ where we consider $s_{1}-1$ and $t_{1}-1 \bmod n+1$. Now for $r \geqslant 2$, let $B_{1}, B_{2} \in \pi_{r-1}$ be the blocks that one merges to obtain $\pi_{r}$ and assume that $\min B_{1}<\min B_{2}$. Define $s_{1}:=\max \left\{i \in B_{1}: i<\min B_{2}\right\} \in B_{1}$ and $t_{1}:=\max B_{2} \in B_{2}$. Let $c\left(i_{r-1}, j_{r-1}\right):=c\left(s_{1}-1, t_{1}-1\right)$. Finally, put $g(\mathcal{C}):=\left\{\left(c\left(i_{\ell}, j_{\ell}\right), \ell\right): \ell \in[k]\right\}$.

We claim that $g(\mathcal{C})$ has no crossing chords. Suppose $\left(c\left(s_{i}, t_{i}\right), i\right)$ and $\left(c\left(s_{j}, t_{j}\right), j\right)$ are crossing chords in $g(\mathcal{C})$ with $i<j$ and $i, j \in[k]$. We further assume that

$$
j=\min \left\{j^{\prime} \in[i+1, k]:\left(c\left(s_{j^{\prime}}, t_{j^{\prime}}\right), j^{\prime}\right) \operatorname{crosses}\left(c\left(s_{i}, t_{i}\right), i\right) \text { in } g(\mathcal{C})\right\} .
$$

We observe that $s_{i}+1, t_{i}+1 \in B_{1}$ for some block $B_{1} \in \pi_{j}$ and that $s_{j}+1, t_{j}+1 \in B_{2}$ for some block $B_{2} \in \pi_{j+1}$. We further observe that $s_{j}+1, t_{j}+1 \notin B_{1}$ otherwise, by the definition of the map $g$, the chords $\left(c\left(s_{i}, t_{i}\right), i\right)$ and $\left(c\left(s_{j}, t_{j}\right), j\right)$ would be noncrossing. Thus $B_{1}, B_{2} \in \pi_{j+1}$ are distinct blocks that cross so $\pi_{j+1} \notin N C^{\mathbb{A}}(n+1)$. We conclude that $g(\mathcal{C})$ has no crossing chords.

Lastly, we show that $g(\mathcal{C})$ has a good labeling. Suppose $c(i, j)$ and $c\left(i, j^{\prime}\right)$ are two chords of $g(\mathcal{C})$ where $c\left(i, j^{\prime}\right)$ is clockwise from $c(i, j)$. By the definition of $g$, chord $c\left(i, j^{\prime}\right)$ was added after chord $c(i, j)$ so the label of chord $c\left(i, j^{\prime}\right)$ is greater than that of $c(i, j)$. Thus $g(\mathcal{C}) \in \hat{\mathcal{D}}_{k, \epsilon}$.

To complete the proof, we show that $g \circ f=1_{\hat{\mathcal{D}}_{k, \epsilon}}$. The proof that $f \circ g$ is the identity map is similar. Let $\hat{d} \in \hat{\mathcal{D}}_{k, \epsilon}$. Then $f(\hat{d})=\left(\pi_{1}=\{\{i\}\}_{i \in[n+1]}, \pi_{2}, \ldots, \pi_{k+1}\right)$ where for any $s \in[k]$ we have

$$
\pi_{s}=\left(\pi_{s-1} \backslash\left\{B_{\alpha}, B_{\beta}\right\}\right) \sqcup\left\{B_{\alpha}, B_{\beta}\right\}
$$

where $i_{s-1}+1 \in B_{\alpha}$ and $j_{s-1}+1 \in B_{\beta}$. Then we have $g(f(\hat{d}))=\left\{c\left(\left(i_{\ell}+1\right)-1,\left(j_{\ell}+1\right)-\right.\right.$ $1), \ell)\}_{\ell \in[k]}=\left\{\left(c\left(i_{\ell}, j_{\ell}\right), \ell\right)\right\}_{\ell \in[k]}$.

Corollary 48. If $\epsilon=(-, \ldots,-)$ or $\epsilon=(+, \ldots,+)$, then the exceptional sequences of $Q_{\epsilon}$ are in bijection with saturated chains in $N C^{\mathbb{A}}(n+1)$ of the form

$$
\left\{\left(\pi_{1}=\{\{i\}\}_{i \in[n+1]}, \pi_{2}, \ldots, \pi_{k+1}\right): \begin{array}{c}
\pi_{j}=\left(\pi_{j-1} \backslash\left\{B_{\alpha}, B_{\beta}\right\}\right) \sqcup\left\{B_{\alpha} \sqcup B_{\beta}\right\} \\
\text { for some } B_{\alpha} \neq B_{\beta} \text { in } \pi_{j-1}
\end{array}\right\} .
$$

Example 49. In Figure 12, we give two examples of the bijection from Theorem 47 with $k=4$. 


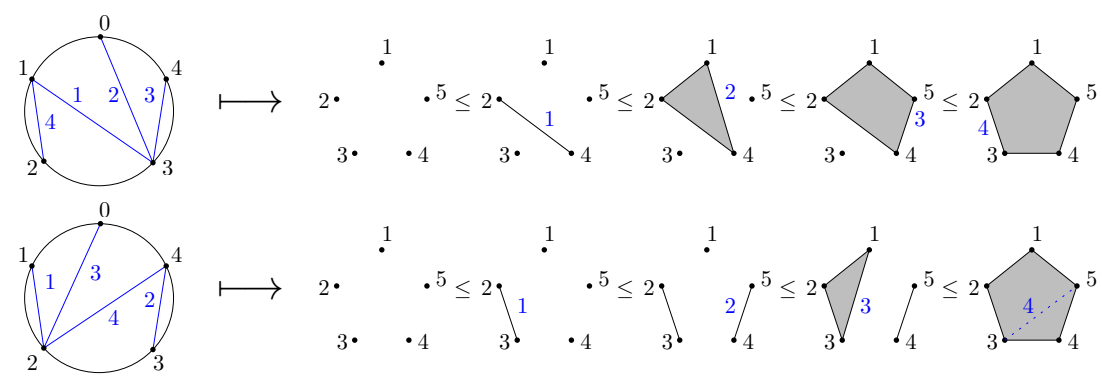

Figure 12: Two labeled strand diagrams and their corresponding maximal chains in $N C^{\mathbb{A}}(5)$.

\section{Acknowledgements}

A. Garver and J. Matherne gained helpful insight through conversations with E. Barnard, J. Geiger, M. Kulkarni, G. Muller, G. Musiker, D. Rupel, D. Speyer, and G. Todorov. A. Garver and J. Matherne also thank the 2014 AMS Mathematics Research Communities program for giving them a stimulating place to work. The authors thank the anonymous referees for their careful comments that helped to greatly improve the exposition.

\section{References}

[Ara99] T. Araya. Exceptional sequences over graded Cohen-Macaulay rings. Math. J. Okayama Univ., 41:81-102, 1999.

[Ara13] T. Araya. Exceptional sequences over path algebras of type $A_{n}$ and noncrossing spanning trees. Algebr. Represent. Theory, 16(1):239-250, 2013.

[ASS06] I. Assem, D. Simson, and A. Skowronski. Elements of the representation theory of associative algebras. Vol. 1, volume 65 of London Mathematical Society Student Texts. Cambridge University Press, Cambridge, 2006. Techniques of representation theory.

[Bes03] D. Bessis. The dual braid monoid. Ann. Sci. École Norm. Sup. (4), 36(5):647683, 2003.

[Bez03] R. Bezrukavnikov. Quasi-exceptional sets and equivariant coherent sheaves on the nilpotent cone. Represent. Theory, 7:1-18 (electronic), 2003.

[BK89] A. I. Bondal and M. M. Kapranov. Representable functors, Serre functors, and reconstructions. Izv. Akad. Nauk SSSR Ser. Mat., 53(6):1183-1205, 1337, 1989.

[CB93] W. Crawley-Boevey. Exceptional sequences of representations of quivers [ MR1206935 (94c:16017)]. In Representations of algebras (Ottawa, ON, 1992), volume 14 of CMS Conf. Proc., pages 117-124. Amer. Math. Soc., Providence, RI, 1993. 
[DWZ10] H. Derksen, J. Weyman, and A. Zelevinsky. Quivers with potentials and their representations II: applications to cluster algebras. J. Amer. Math. Soc., 23(3):749-790, 2010.

[GM15] A. Garver and J. Matherne. A combinatorial model for exceptional sequences in type A. DMTCS (Disc. Math. \& Theor. Comp. Sci.) proc., FPSAC'15:393404, 2015.

[GY02] I. Goulden and A. Yong. Tree-like properties of cycle factorizations. J. Combin. Theory Ser. A, 98(1):106-117, 2002.

[Hap88] D. Happel. Triangulated categories in the representation theory of finitedimensional algebras, volume 119 of London Mathematical Society Lecture Note Series. Cambridge University Press, Cambridge, 1988.

[HK16] A. Hubery and H. Krause. A categorification of non-crossing partitions. $J$. Eur. Math. Soc. (JEMS), 18(10):2273-2313, 2016.

[IO13] K. Igusa and J. Ostroff. Mixed cobinary trees. J. Algebra Appl., 17(9):1850170, 2018.

[IS10] K. Igusa and R. Schiffler. Exceptional sequences and clusters. J. Algebra, 323(8):2183-2202, 2010.

[IT09] C. Ingalls and H. Thomas. Noncrossing partitions and representations of quivers. Compos. Math., 145(6):1533-1562, 2009.

[Kel12] B. Keller. Cluster algebras and derived categories. In Derived categories in algebraic geometry, EMS Ser. Congr. Rep., pages 123-183. Eur. Math. Soc., Zürich, 2012.

[Kel13] B. Keller. Quiver mutation and combinatorial DT-invariants. FPSAC 2013 Abstract, 2013.

[Mel04] H. Meltzer. Exceptional vector bundles, tilting sheaves and tilting complexes for weighted projective lines. Mem. Amer. Math. Soc., 171(808):viii+139, 2004.

[NC15] A. Nájera Chávez. On the c-vectors of an acyclic cluster algebra. Int. Math. Res. Not. IMRN, (6):1590-1600, 2015.

[ONA $\left.{ }^{+} 13\right]$ M. Obaid, K. Nauman, W. S. M. Al-Shammakh, W. Fakieh, and C. M. Ringel. The number of complete exceptional sequences for a Dynkin algebra. Colloq. Math., 133(2):197-210, 2013.

[Rin94] C. M. Ringel. The braid group action on the set of exceptional sequences of a hereditary Artin algebra. In Abelian group theory and related topics (Oberwolfach, 1993), volume 171 of Contemp. Math., pages 339-352. Amer. Math. Soc., Providence, RI, 1994.

[Rud90] A. N. Rudakov. Exceptional collections, mutations and helices. In Helices and vector bundles, volume 148 of London Math. Soc. Lecture Note Ser., pages 1-6. Cambridge Univ. Press, Cambridge, 1990. 
[Sei01] U. Seidel. Exceptional sequences for quivers of Dynkin type. Comm. Algebra, 29:1373-1386, 2001.

[ST13] D. Speyer and H. Thomas. Acyclic cluster algebras revisited. In Algebras, quivers and representations, volume 8 of Abel Symp., pages 275-298. Springer, Heidelberg, 2013. 